\title{
Correspondence
}

\section{Throughput Maximization for a Buffer-Aided Successive $2 \quad$ Relaying Network Employing Energy Harvesting}

3

\author{
Shruti Gupta, Rong Zhang, and Lajos Hanzo
}

4 Abstract-Energy harvesting (EH)-assisted nodes are capable of sig5 nificantly prolonging the lifetime of future wireless networks, provided 6 that they rely on appropriate transmission policies, which accommo7 date the associated stochastic energy arrival. In this paper, a successive8 relaying-based network using rechargeable source and relay nodes having 9 limited buffers for both their energy and data storage is considered. The 10 maximization of the network throughput with noncausal knowledge of en11 ergy arrivals by the deadline $\boldsymbol{T}$ is formulated as a nonconvex optimization 12 problem, and it is solved using the interior-point optimization (IPOPT) 13 method. The performance of the low-complexity suboptimal scheme was 14 found to reach its maximum when the two phases of the successive relaying 15 protocol have equal duration. The optimal and suboptimal schemes are 16 capable of achieving up to $92 \%$ and $88 \%$ of the throughput performance of 17 the benchmark scheme. The suboptimal scheme's throughput performance 18 is consistently about $90 \%$ of that of the optimal scheme. For asymmetric 19 data (or energy) buffer sizes, it was found that the throughput performance 20 depends on the total (i.e., collective) data (or energy) buffer capacity 21 available in the network and not just on the smallest data buffer.

22 Index Terms-Author, please supply index terms/keywords for your 23 paper. To download the IEEE Taxonomy go to http://www.ieee.org/ 24 documents/taxonomy_v101.pdf.

\section{INTRODUCTION}

26 Cooperative communication is capable of attaining significant 27 throughput and reliability improvements, where the source node $(S N)$ 28 and cooperating relay nodes $(R N)$ expend their energy while process29 ing and transmitting the signal to the destination node $(D N)$. The 30 nodes are typically powered through precharged batteries, but once 31 these batteries are drained, the nodes become dysfunctional [1], [2]. An 32 emerging solution to this vexed problem is the use of energy harvesting 33 (EH) [1]-[3], which has to be capable of accommodating the random 34 arrivals of energy and its storage at the nodes [4].

35 Hence, EH communication systems have been studied under dif36 ferent network models. In [5]-[7], a single-user EH system was char37 acterized, where beneficial power-allocation strategies were designed 38 under the corresponding EH constraints. This was further extended to 39 the design of an EH-aided broadcast channel in [8] and [9] and to 40 two-way orthogonal frequency-division multiplexing communications 41 [10]. In [8], Yang et al. defined the cutoff power levels for each 42 user to allocate the optimal power to them, whereas in [9], Kuan 43 et al. analyzed the tradeoff between the achievable reliability and 44 throughput for broadcast transmissions relying on erasure codes for $45 \mathrm{EH}$ sensors. In [10], the receiver is designed both for simultaneously

Manuscript received January 28, 2015; revised June 3, 2015 and July 30, 2015; accepted September 8, 2015. This work was supported by the European Research Council under the Advanced Fellow Grant. The review of this paper was coordinated by Dr. L. Zhao.

The authors are with the School of Electronics and Computer Science, University of Southampton, Southampton SO17 1BJ, U.K. (e-mail: sg7g12@ ecs.soton.ac.uk;rz@ecs.soton.ac.uk; lh@ecs.soton.ac.uk).

Color versions of one or more of the figures in this paper are available online at http://ieeexplore.ieee.org.

Digital Object Identifier 10.1109/TVT.2015.2477808

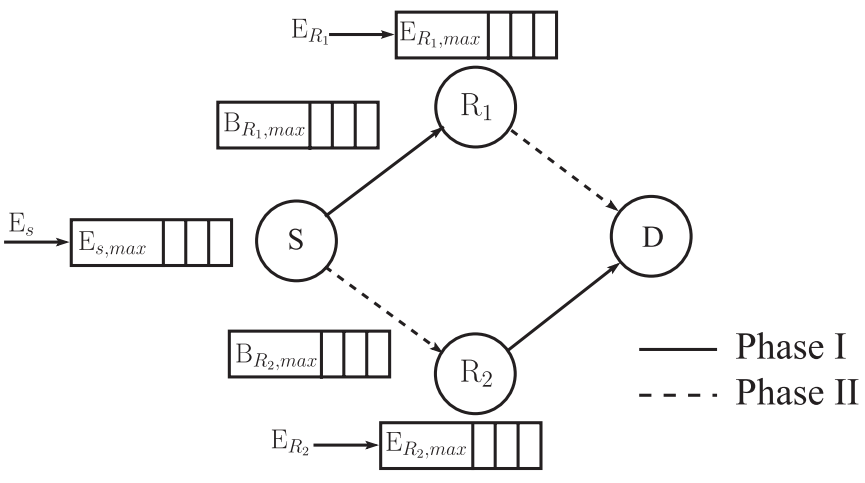

Fig. 1. Successive relaying network where EH nodes are equipped with finite buffer for both energy and data storage.

processing information and for harvesting energy from the received 46 desired signal, as well as jamming interference a through power 47 splitter. In recent years, cooperative networks have also been studied 48 in the context of EH at the RNs and/or the $S N$ [1]-[3], [11]-[13]. 49 Specifically, in [1], Medepally and Mehta investigated the benefits 50 of relay selection relying on multiple EH amplify-and-forward RNs, 51 whenever they have sufficient energy for transmission. By contrast, 52 in [2], information-buffer-aided link activation was used, which was 53 controlled both by the quality of the links and by the amount of 54 energy buffered at these nodes. Two-hop networks relying either on a 55 single or on a pair of parallel RNs using a successive relaying protocol 56 were investigated to quantify the benefits of both multiple relays and 57 of EH on the average throughput of the system in [3]. In [11], the 58 authors derived the optimal achievable rates for an $\mathrm{EH}$ system in the 59 context of two-way relaying employing different relaying strategies. 60 Furthermore, a similar two-way EH relay system employing time- 61 division broadcasting and multiple access broadcasting, which was 62 subjected to channel state uncertainty, was considered in the context 63 of joint energy and transmit time allocation in [12]. Utilizing the struc- 64 ture of a specific problem and the generalized optimality principle, 65 in [13], a new algorithm for constrained utility maximization problems 66 encountered in a cooperative network of wireless sensor nodes is 67 formulated.

Against this background, we consider a successive relaying model, 69 which is capable of mimicking a full-duplex (FD) $R N$, despite relying 70 on a pair of half-duplex (HD) RNs, which are activated alternately in 71 their transmitter and receiver modes to create a virtual FD relay. This 72 HD regime reduces the complexity of the FD system, since the FD 73 $R N$ would require high-complexity interference cancellation at the 74 receiver. In contrast to [3], our model relies on the realistic constraint 75 that $\mathrm{EH}$ nodes $\left(S N, R N_{1}, R N_{2}\right)$ have a finite energy storage capacity 76 and that the RNs also have limited data buffers for storing the source 77 data. We first formulate an optimization problem for the throughput 78 maximization of our successive-relaying-aided network in Fig. 1 hav- 79 ing finite buffers, as well as relying on the idealized noncausal 80 knowledge of the energy arrivals at all EH nodes. Then, using the 81 interior-point optimization method (IPOPT), the optimization problem 82 is solved for both optimal and suboptimal schemes, and finally, we 83 quantify the effect of buffer sizes on the throughput of the network 84 based on both schemes. While proof-of-concept studies are indeed 85 
86 valuable, the ultimate purpose of most engineering studies is to attempt 87 a real-world implementation of the proposed techniques. Through this 88 study, we aimed to take the valuable proposals in [3] a step closer to 89 its real-world deployment. Explicitly, the novelty of this contribution 90 is given as follows: 1) We define a practical successive relaying model 91 constrained by both limited energy and data storage buffers at the EH 92 nodes, which dispenses with the idealized simplifying assumption of 93 having infinite buffers [3]; 2) we formulate the optimal transmission 94 policy; and 3) we propose a suboptimal transmission scheme capable 95 of approaching the performance of its optimal counterpart at signif96 icantly reduced complexity, which is achieved at the expense of a 97 marginally degraded performance. In our study, we also consider the 98 scenario of asymmetric fading, energy, and data buffers. This paper is 99 organized as follows. In Section II, our system model is presented, 100 which is followed by the formulation of our optimization problem 101 in Section III. Our results are discussed in Section IV, whereas our 102 conclusions are offered in Section V.

\section{SYSTEM MODEL}

104 We consider the successive relaying technique of [3] having two 105 phases, where the RNs assist the SN's transmission to the $D N$, as 106 shown in Fig. 1. In Phase I in Fig. 1, the $S N$ transmits to $R N_{1}$, 107 whereas $R N_{2}$ simultaneously transmits to the $D N$. By contrast, in 108 Phase II in Fig. $1, S N$ and $R N_{1}$ transmit simultaneously both to $109 R N_{2}$ and to $D N$, respectively. Thus, the $S N$ is always transmitting, 110 whereas the $D N$ is always receiving during the process. It is assumed 111 that there is no direct link between $S N-D N$ and $R N_{1}-R N_{2}$, as well 112 as that these are decode-and-forward (DF) HD RNs that are located 113 sufficiently far apart from each other to avoid any interference. We 114 assume that $S N, R N_{1}$, and $R N_{2}$ harvest energy from the environment 115 and have finite energy buffers that can store a maximum of $E_{S, \max }$, $116 E_{R 1, \max }$, and $E_{R 2, \max }$ units, respectively, whereas $R N_{1}$ and $R N_{2}$ 117 are also equipped with data buffers of $B_{R 1 \text {, max }}$ and $B_{R 2, \max }$ packets, 118 respectively. For ease of exposition, we merge the energy arrival events 119 at all the $\mathrm{EH}$ nodes into a single time series $\left(t_{0}, t_{1}, \ldots, t_{K}\right)$ by consid120 ering zero amount of energy arrivals at the nodes that do not harvest 121 energy at some instant $t_{k}$. More explicitly, the EH processes at the EH 122 nodes are independent of each other. In other words, the energy arrival 123 instances of a node may be different from those of the other nodes. 124 For example, assume that an energy arrival occurred at node $R N_{1}$ at 125 some instant $t_{k}$, whereas there was no energy arrival at the other nodes 126 ( $S$ and $R N_{2}$ ) at the time instant $t_{k}$. In our mathematical analysis, 127 we assumed that at time instant $t_{k}$, nodes $S$ and $R N_{2}$ harvested 128 zero amount of energy. We set $t_{0}=0$ and $t_{K}=T$. We represent the 129 amount of energy harvested at $S N, R N_{1}$, and $R N_{2}$ at time instant $t_{k}$ 130 as $E_{S, k}, E_{R 1, k}$, and $E_{R 2, k}$ units, respectively, for $k=0,1, \ldots K-1$. 131 The time interval between the two consecutive energy arrivals is 132 termed as an epoch, whose length is defined as $\tau_{k}=t_{k}-t_{k-1}$. The 133 complex-valued channel gains are considered to be constant through134 out the communication process preceding the deadline. The channel 135 gain between the nodes $L$ and $M$ is denoted by $H_{L M}$, where we have $136 L \in\left\{S N, R_{1} N, R_{2} N\right\}$ and $M \in\left\{R N_{1}, R N_{2}, D N\right\}$.

137 We consider the throughput maximization problem under the ide138 alized simplifying assumption of having prior knowledge about the 139 energy arrivals at all the EH nodes before the commencement of 140 the communication process. We assume that the energy expended at 141 the nodes is only the transmission energy and that perfect "capacity142 achieving" codes are used, which facilitate operation exactly at the 143 Shannon capacity, thus determining the rate versus power relationship 144 of a given link, which is given by

$$
r[p(t)]=\log _{2}[1+H p(t)]
$$

where $H$ is the channel gain of the link, and $p(t)$ is the transmission 145 power of the node at time $t$. As a result of energy arrivals over time and 146 as a benefit of the energy storage capacity at the nodes, any feasible 147 transmission policy should satisfy following constraints.

1) Energy causality constraint: The total energy expended by a 150 node during its transmission session should not exceed the total 151 energy harvested by that node until that time.

2) Energy overflow constraint: The energy exceeding the storage 153 capacity of the energy buffer at the node is lost owing to 154 overflow.

3) Data causality constraint: The total data transmitted by a node 156 during the process should not exceed the total data received by 157 that node until that time.

4) Data overflow constraint: The amount of data exceeding the 159 storage capacity of data buffer is lost due to overflow.

\section{PRoblem Formulation}

Here, we first stipulate some properties of the optimal transmission 162 policy in the following two lemmas, which will be used to formulate 163 the throughput maximization problem for the system in Fig. 1. The 164 proof of these lemmas is provided in Appendixes A and B. 165

Lemma 1: The transmission rate/power of a node is constant be- 166 tween two consecutive energy arrivals but potentially changes when 167 new energy arrives at the node [3].

Lemma 2: The feasible transmission policy ensures that the relays 169 are always on without decreasing the throughput of the system [3]. 170

Based on Lemmas 1 and 2, we can characterize the optimal policy in 171 the following way. There is a constant transmission rate for the pair of 172 nodes between consecutive energy arrivals according to the optimal 173 policy, as formulated in Lemma 1. Therefore, we assume that the 174 transmission power of $S N$ during Phases I and II in Fig. 1 in an epoch 175 is constant and given by $p_{S I, k}$ and $p_{S I I, k}$, respectively. Similarly, 176 the transmission power of $R N_{1}$ and $R N_{2}$ is denoted by $p_{R_{1}, k}$ and 177 $p_{R_{2}, k}$, respectively. Lemma 2 implies that we restrict our attention 178 to the specific transmission policies, where both $R N_{1}$ and $R N_{2}$ are 179 always on for the sake of defining a feasible transmission policy. Thus, 180 we assume that the total transmission time between $S N-R N_{1}$ and 181 $R N_{2}-D N$ is the same and denote this duration of Phase I between 182 the time instants $t_{k-1}$ and $t_{k}$ by $L_{I, k}$. Similarly, we assume the same 183 transmission time between $S N-R N_{2}$ and $R N_{1}-D N$ in Phase II, 184 which is denoted by $L_{I I, k}, k=1,2, \ldots, K$. Finally, we identify 185 the optimal transmission policy that defines which particular node 186 transmits and when, along with the specific power allocation of each 187 node. We then define a suboptimal scheme, where the duration of each 188 phase of successive relaying is fixed to a particular ratio.

\section{A. Optimal Transmission Policy}

Let us now define the optimization problem of maximizing the 191 system throughput by the deadline $T$. Since $R N_{2}$ initially has no data 192 in Phase I in Fig. 1, it is assumed without loss of generality that it 193 starts transmission by delivering $\epsilon>0$ amount of dummy information 194 to $D N$, where $\epsilon$ is sufficiently small to be ignored for our throughput 195 optimization problem. Upon scheduling the two phases in succession, 196 it is ensured that there is no further throughput loss for the system. 197 In other words, at the beginning of transmission, $R N_{2}$ possesses no 198 data from $S$ that can be transmitted to $D N$; hence, it commences its 199 transmission with $\epsilon$ dummy packets. However, subsequently, the trans- 200 mission phases occur in immediate succession without any interval. 201 This ensures that there is no need to send dummy packets, and thus, 202 no further loss of system throughput is imposed. Similar assumptions 203 were also made in [3]. We first define the throughput of the nodes 204 
205 in different phases based on the rate versus power relationship (1) 206 mentioned in Section II as

$$
\begin{aligned}
\alpha_{R 1, k} & =L_{I I, k} \log _{2}\left(1+H_{R 1 D} p_{R 1, k}\right) \\
\alpha_{R 2, k} & =L_{I, k} \log _{2}\left(1+H_{R 2 D} p_{R 2, k}\right) \\
\alpha_{S I, k} & =L_{I, k} \log _{2}\left(1+H_{S R 1} p_{S I, k}\right) \\
\alpha_{S I I, k} & =L_{I I, k} \log _{2}\left(1+H_{S R 2} p_{S I I, k}\right) .
\end{aligned}
$$

207 Now, the optimization problem is defined over $L_{I, k}, L_{I I, k}, \alpha_{S I, k}$, $208 \alpha_{S I I, k}, \alpha_{R 1, k}$, and $\alpha_{R 2, k},(3 \mathrm{a})-(3 \mathrm{~m})$, shown at the bottom of the page. 209 Note that when (3h)-(3i) are evaluated at $k=K$, the total amount 210 of data delivered to $D N$ is equal to the amount of data transferred by $R N_{1}$ and $R N_{2}$; hence, the throughput maximization problem 211 corresponds to the maximization of the amount of data transmitted 212 by both the RNs, as formulated in (3a). The problem in (3) is a non- 213 convex optimization problem, owing to the nonconvex energy storage 214 constraints defined in (3e)-(3g), which can be efficiently solved using 215 the IPOPT method, as given in Appendix C.

\section{B. Suboptimal (Alternate) Transmission Policy}

In this scheme, we set the duration of Phase I in Fig. 1 to be equal 218 to $\eta \%$; of the length of an epoch, i.e., we have

$$
L_{I, k}=\frac{\eta}{100} \tau_{k}, \quad L_{I I, k}=\tau_{k}-\frac{\eta}{100} \tau_{k} .
$$

$\operatorname{maximize} \quad \sum_{k=1}^{K} \alpha_{R 1, k}+\alpha_{R 2, k}$

subject to

Energy causality constraints (constraint 1 in Section II) at $S N, R N_{1}$, and $R N_{2}$ :

$$
\begin{aligned}
& \sum_{j=1}^{k} \frac{L_{I, j}}{H_{S R 1}}\left(2\left(\frac{\alpha_{S I, j}}{L_{I, j}}\right)-1\right)+\frac{L_{I I, j}}{H_{S R 2}}\left(2^{\left(\frac{\alpha_{S I I, j}}{L_{I I, j}}\right)}-1\right) \leq \sum_{j=0}^{k-1} E_{S, j} \quad \forall k \\
& \sum_{j=1}^{k} \frac{L_{I I, j}}{H_{R 1 D}}\left(2^{\left(\frac{\alpha_{R I, j}}{L_{I I, j}}\right)}-1\right) \leq \sum_{j=0}^{k-1} E_{R 1, j} \quad \forall k \\
& \sum_{j=1}^{k} \frac{L_{I, j}}{H_{R 2 D}}\left(2^{\left(\frac{\alpha_{R 2, j}}{L_{I, j}}\right)}-1\right) \leq \sum_{j=0}^{k-1} E_{R 2, j} \quad \forall k .
\end{aligned}
$$

Energy overflow constraints (constraint 2 in Section II) at $S N, R N_{1}$, and $R N_{2}$ :

$$
\begin{aligned}
& \sum_{j=0}^{k} E_{S, j}-\sum_{j=1}^{k} \frac{L_{I, j}}{H_{S R 1}}\left(2\left(\frac{\alpha_{S I, j}}{L_{I, j}}\right)-1\right)+\frac{L_{I I, j}}{H_{S R 2}}\left(2\left(\frac{\alpha_{S I I, j}}{L_{I I, j}}\right)-1\right) \leq E_{S, \max } \quad \forall k \\
& \sum_{j=0}^{k} E_{R 1, j}-\sum_{j=1}^{k} \frac{L_{I I, j}}{H_{R 1 D}}\left(2\left(\frac{\alpha_{R 1, j}}{L_{I I, j}}\right)-1\right) \leq E_{R 1, \max } \quad \forall k \\
& \sum_{j=0}^{k} E_{R 2, j}-\sum_{j=1}^{k} \frac{L_{I, j}}{H_{R 2 D}}\left(2\left(\frac{\alpha_{R 2, j}}{L_{I, j}}\right)^{i}-1\right) \leq E_{R 2, \max } \quad \forall k .
\end{aligned}
$$

Data causality constraints (constraint 3 in Section II) at $R N_{1}$ and $R N_{2}$ :

$$
\begin{array}{ll}
\sum_{j=1}^{k} \alpha_{R 1, j} \leq \sum_{j=1}^{k} \alpha_{S I, j} & \forall k \\
\sum_{j=1}^{k} \alpha_{R 2, j} \leq \sum_{j=1}^{k} \alpha_{S I I, j} & \forall k .
\end{array}
$$

Data overflow constraints (constraint 4 in Section II) at $R N_{1}$ and $R N_{2}$ :

$$
\begin{array}{ll}
\sum_{j=1}^{k} \alpha_{S I, j}-\sum_{j=1}^{k-1} \alpha_{R 1, j} \leq B_{R 1, \max } & \forall k \\
\sum_{j=1}^{k} \alpha_{S I I, j}-\sum_{j=1}^{k-1} \alpha_{R 2, j} \leq B_{R 2, \max } & \forall k .
\end{array}
$$

Half duplex constraint due to the HD relays $R N_{1}$ and $R N_{2}$ :

$L_{I, k}+L_{I I, k} \leq \tau_{k} \quad \forall k$.

Feasibility constraints at $S N, R N_{1}$ and $R N_{2}$ :

$\alpha_{S I, k} \geq 0, \quad \alpha_{S I I, k} \geq 0, \quad \alpha_{R 1, k} \geq 0 ; \alpha_{R 2, k} \geq 0, \quad L_{I, k} \geq 0, \quad L_{I I, k} \geq 0 \quad \forall k$ 


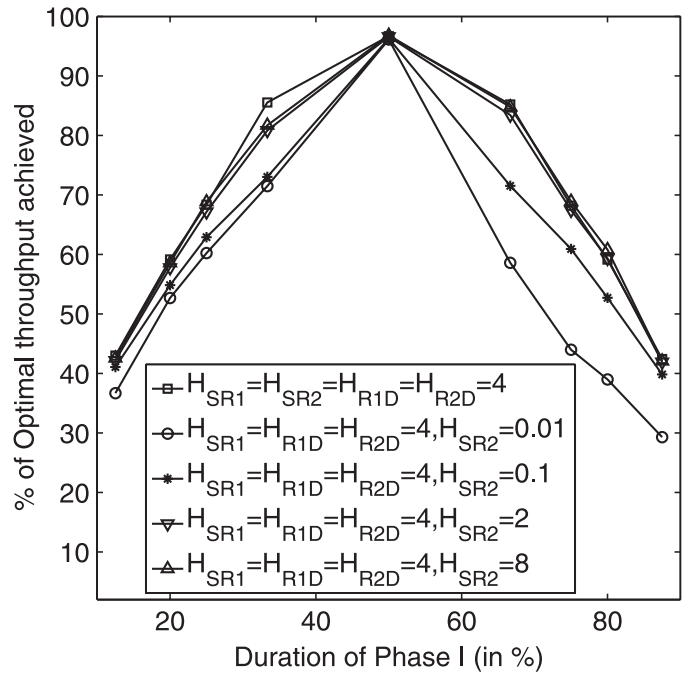

Fig. 2. Relation between percentage of optimal throughput achieved for varying duration of Phase I occurring in an EH epoch with sufficient energy and data buffer sizes (5 and 2, respectively) for different settings of channel gains.

220 Using (4), the optimization problem is relaxed for this suboptimal 221 scheme and can be reformulated by omitting (31) from (3). This is 222 again a nonconvex optimization problem; hence, it may be solved 223 using the IPOPT method. This scheme is termed suboptimal, since 224 the duration of the phases has been deliberately fixed for the sake of 225 reducing the complexity ${ }^{1}$ of the optimization problem.

\section{Performance Results}

227 Here, we evaluate the performance of the proposed buffer-aided 228 successive relaying system relying on offline power allocation in 229 terms of the optimal throughput achieved by the deadline of $T=$ $23010 \mathrm{~s}$. We assume that the EH process of both the $S N$ and the RNs 231 independently takes values from $\left[0, E_{\max }=5\right]$ units, where the energy 232 is uniformly distributed under an exponential inter-arrival time at 233 a rate of $\lambda_{e}=5$ units/s. The deterministic channel gains are set to 234 the values $H_{S R 1}=H_{S R 2}=H_{R 1 D}=H_{R 2 D}=4$, except otherwise 235 mentioned. Our results quantify the throughput of the system as a 236 function of both data and energy buffer capacity for both optimal 237 and suboptimal schemes that are benchmarked against the infinite238 storage-based optimal scheme defined in [3]. Our benchmark scheme 239 of [3] is insensitive to the buffer sizes, since it considers infinite 240 storage capacities at all the EH nodes for both energy and data, thereby 241 providing an upper bound to our proposed system.

242 The percentage duration of Phases I and II in Fig. 1 is not fixed 243 for the optimal scheme, whereas they have been fixed to a specific 244 ratio for the suboptimal scheme for the sake of complexity reduction. 245 Hence, our first goal was to identify the specific ratio of the durations 246 of Phases I and II that would maximize the throughput of the sub247 optimal scheme. Fig. 2 shows the specific percentage of the optimal 248 throughput, which was actually achieved by varying the proportion of 249 the Phase I duration $\left(L_{I}\right)$ in each of the EH epochs, along with the 250 symmetric $\left(H_{S R 1}=H_{S R 2}=H_{R 1 D}=H_{R 2 D}=4\right)$ and asymmetric 251 settings of the channel fading gain for $S N-R N_{2}$. The performance 252 of the suboptimal scheme peaks when the durations of both phases 253 are equal. For the other scenarios, the throughput is lower, because the 254 amount of data transmitted between $S N$ and $D N$ is limited by the 255 shorter phase. It is shown in Fig. 2 that, as the duration of the shorter 256 phase increases, the throughput also increases. It is interesting to note

${ }^{1}$ The complexity analysis of both schemes is beyond the scope of this paper.

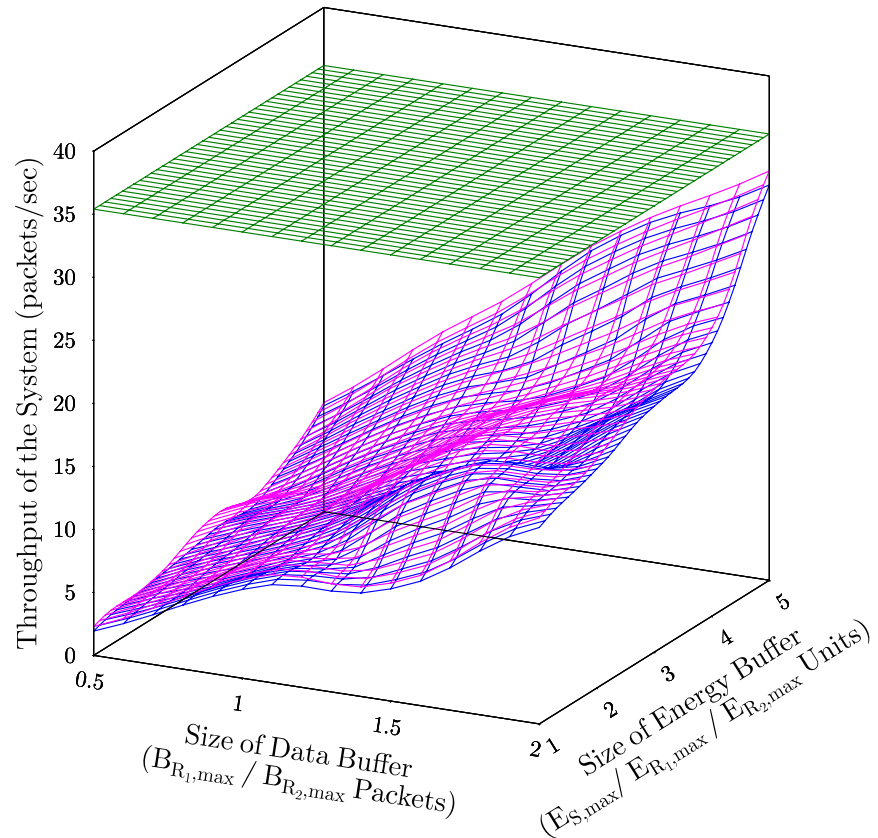

Fig. 3. Impact of the energy and data buffer sizes at all the EH nodes on the throughput of the system by the deadline $T$. The constant green surface represents the throughput of the benchmark scheme [3], whereas the pink and blue surfaces depict our optimal and suboptimal transmission policies, respectively.

that in the scenarios of very low channel gain, i.e., for $H_{S R 2}=0.01257$ and $H_{S R 2}=0.1$, there exists asymmetry in the throughput achieved 258 by the system. The reason behind this trend is that when the duration 259 of Phase I is higher than that of Phase II, the channel gain of path 260 $S N-R N_{2}$ limits the amount of data that can be otherwise transmitted 261 to $R N_{2}$. As shown in Fig. 2, when the duration of Phase I is $50 \%$ of 262 the $\mathrm{EH}$ epoch, the suboptimal scheme achieves approximately $97 \%$ of 263 the optimal scheme's throughput. Hence, in the following discussions, 264 we consider a suboptimal scheme, where the duration of each phase is 265 $50 \%$ of the epoch duration.

The 3-D characterization of the system in Fig. 1 is provided in 267 Fig. 3. Specifically, Fig. 3 shows the overall throughput of the system 268 as a function of the size of both energy and data buffers at the EH 269 nodes. It can be clearly observed that, with the increase in the size of 270 buffers at the EH nodes, the throughput of our proposed schemes 271 improve owing to increased availability of energy and data storage 272 capacity at the EH nodes, supporting a larger amount of data trans- 273 mission to $D N$. However, the throughput of the benchmark scheme 274 [3] is constant, i.e., independent of the buffer sizes, as it relies on the 275 idealized settings where EH nodes possess infinite energy and data 276 storage capacity. Moreover, our optimal scheme performs only mar- 277 ginally better than our less complex suboptimal scheme, because the 278 duration of each phase is fixed in the suboptimal scheme. This would, 279 in turn, result in limiting the amount of data that can be transmitted to 280 $D N$ during successive relaying phases. To closely analyze the impact 281 of the energy and data buffer capacities at the EH nodes on the overall 282 system throughput, we present the 2-D curves corresponding to the 283 individual analysis of the energy buffer size while keeping the data 284 buffer size constant, and vice versa.

The results in Fig. 4 show the throughput of the system against 286 the size of the battery in the presence of sufficient, insufficient, and 287 asymmetric data buffer sizes for both optimal and suboptimal schemes. 288 As expected, upon increasing the battery size, the throughput of the 289 system is improved, owing to the availability of increased amount of 290 


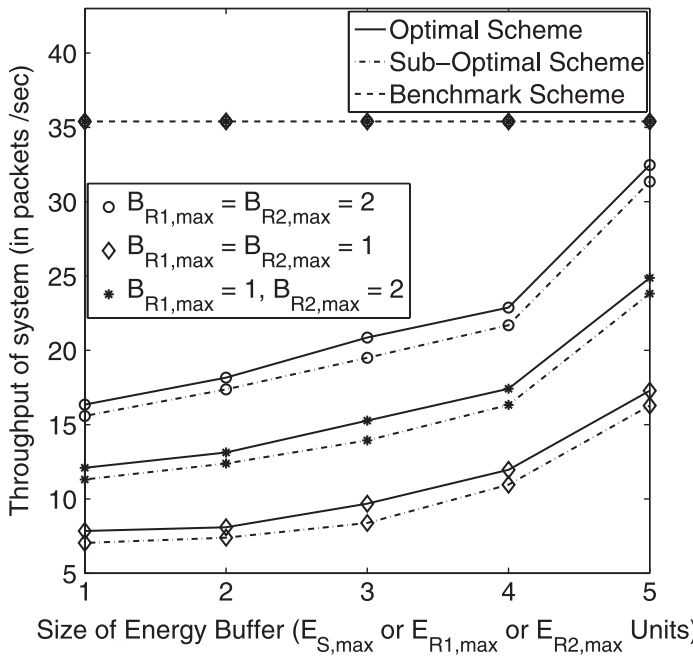

Fig. 4. Impact of energy buffer size at all the EH nodes with sufficient (two packets), insufficient (one packet), and asymmetric data buffer capacity at the RNs on the throughput of the system by the deadline $T$.

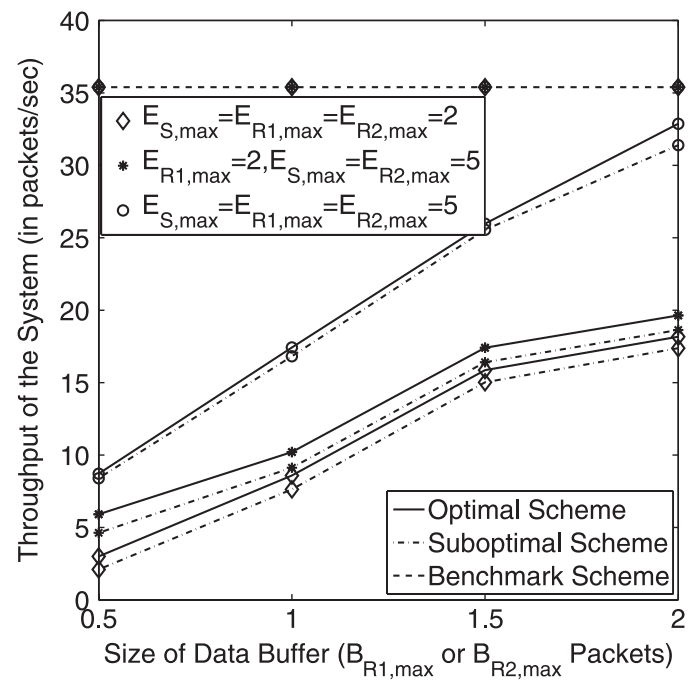

Fig. 5. Impact of data buffer size at the RNs with sufficient (five units), insufficient (two units), and asymmetric battery capacities at EH nodes over throughput of the system by the deadline $T$.

291 energy (due to the increase in buffer size) for transmission. Moreover, 292 it can be observed that for sufficient (or insufficient) data storage, 293 our optimal system is capable of achieving 92\% (or 50\%) of the 294 benchmark scheme's throughput performance [3], whereas our sub295 optimal scheme performs slightly worse than the optimal scheme, 296 reaching $88 \%$ (or $46 \%$ ) of the benchmark system's throughput value 297 in [3], when the battery capacity of the EH nodes is sufficiently high $298\left(E_{S, \max }=E_{R_{1}, \max }=E_{R_{2}, \max }=5\right.$ units $)$. Furthermore, for asym299 metric settings having unequal data buffers at $R N_{1}$ and $R N_{2}$, the 300 throughput becomes lower than that for sufficiently large storage, since $301 R N_{1}$ is now acting as a bottleneck, preventing the flow of data to $D N$. 302 On the other hand, for this asymmetric setting, the throughput becomes 303 higher than that for insufficient storage, since the node $R N_{2}$ has a 304 higher data storage capacity, thereby supporting a higher data rate to $305 D N$. The suboptimal scheme's throughput performance was $95.2 \%$, $30690.7 \%$, and $93.7 \%$ of that of the optimal scheme for the scenarios of 307 sufficient, insufficient, and asymmetric data buffers, respectively.

308 Similarly, Fig. 5 shows the throughput of the system as a function of 309 the data buffer size at the RNs with sufficient, insufficient, and asym-

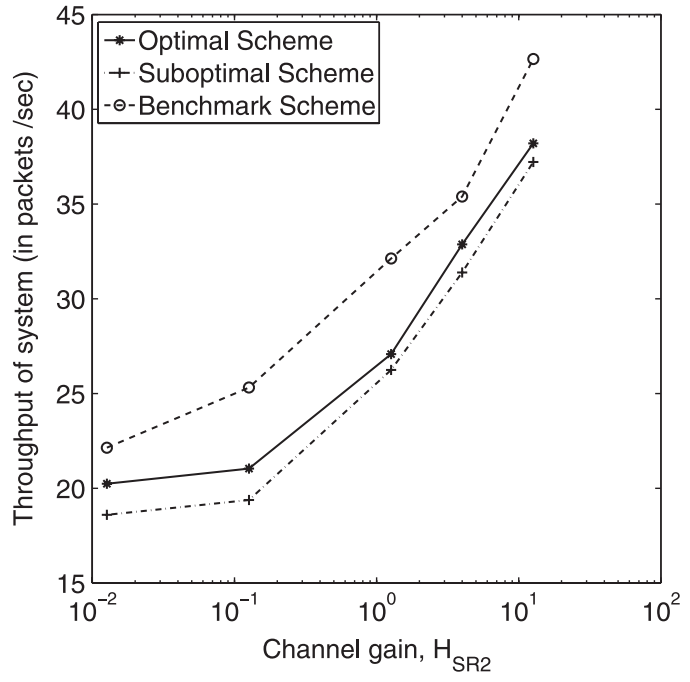

Fig. 6. Impact of asymmetric fading from $S$ to $R N_{2}$ for sufficient battery and data buffer capacities (five units and two packets, respectively) at $\mathrm{EH}$ nodes on throughput of the system by the deadline $T$.

metric energy buffer sizes for both optimal and suboptimal schemes. 310 It is clearly demonstrated that as the size of the data buffer increases, 311 the amount of data successfully transmitted to the $D N$ also increases 312 for both schemes, indicating that the optimal and suboptimal schemes 313 have quite similar performance. The reason behind this trend is the 314 reduction of overflowing data buffers owing to the larger capacities of 315 these buffers at the RNs. Furthermore, for sufficient (or insufficient) 316 battery capacities, our optimal system having finite buffers is capable 317 of achieving $92 \%$ (or 52\%) of the throughput compared with our 318 suboptimal scheme that performs comparably, since it achieves $88 \% 319$ (or 49\%) of the benchmark system's throughput [3] for the maximum 320 data buffer size of $B_{R_{1}, \max }=B_{R_{2} \text {, max }}=2$ packets. Furthermore, for 321 asymmetric settings having unequal energy buffers at $R N_{1}$ and $R N_{2}, 322$ the throughput becomes lower than that for a sufficiently large storage, 323 since $R N_{1}$ is low on energy, hence preventing the flow of data to $D N .324$ On the other hand, for this asymmetric setting, the throughput becomes 325 higher than that for insufficient storage, since the node $R N_{2}$ has a 326 higher energy storage capacity, consequently supporting a higher data 327 rate to $D N$. Moreover, the suboptimal scheme achieves $96.7 \%, 87.3 \%, 328$ and $94.2 \%$ of the throughput of our optimal scheme for sufficient, 329 insufficient, and asymmetric energy buffers, respectively.

330

Fig. 6 shows the throughput of the system as a function of the asym- 331 metric channel gain of the $S N-R N_{2}$ path $\left(H_{S R 2}\right)$ for the scenario of 332 having a sufficiently high data and energy buffer size at the EH nodes, 333 where all other channel gains are set to $H_{S R 1}=H_{R 1 D}=H_{R 2 D}=4.334$ It can be clearly seen that as the channel gain $H_{S R 2}$ increases, the 335 throughput of the system increases for all the schemes owing to the 336 rate-power relationship mentioned in (1). This means that as the value 337 of the channel gain increases, the amount of data transmitted from 338 $S N$ to $R N_{2}$ increases, and so does the amount of data reaching the 339 $D N$, hence, also increasing the overall throughput of the system. As 340 expected, the benchmark scheme represents the upper bound of the 341 system's throughput for an asymmetric setting of the channel gain, 342 as it relies on the idealized assumptions of infinite data and energy 343 storage capacities at the EH nodes. However, our optimal scheme 344 performs better than the suboptimal scheme owing to the fixed duration 345 of phases in the successive relaying protocol of the latter scheme. $\quad 346$

In Fig. 7, we considered the throughput of the system as a function 347 of the data buffer capacity at the RNs for the scenario of asymmetric 348 channel gains and asymmetric energy buffer capacity. Explicitly, we 349 


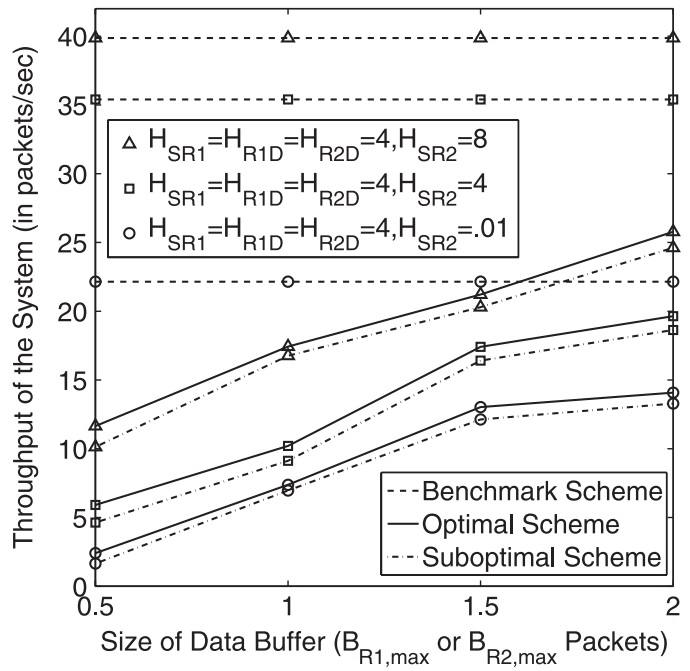

Fig. 7. Impact of data buffer size at the RNs with asymmetric channel gains and battery capacities $\left(E_{S, \max }=E_{R 2, \max }=5\right.$ units, and $E_{R 1, \max }=$ 2 units) at EH nodes over throughput of the system by the deadline $T$.

350 have used $E_{S, \max }=E_{R 2, \max }=5$ units and $E_{R 1, \max }=2$ units at the $351 \mathrm{EH}$ nodes. The benchmark scheme provides an upper bound for our 352 proposed schemes and has a constant throughput, since it is unaffected 353 by the data and energy buffer capacity at the EH nodes. Interestingly, 354 the throughput of the system improves upon increasing the value of the 355 channel gains, which becomes explicit by observing the rate-power 356 relationship of (1). Moreover, the asymmetric setting of energy buffers 357 at the EH nodes of the proposed scheme results in limiting the 358 throughput achieved by the system, because $R N_{1}$ is acting as the 359 bottleneck owing to the low energy buffer capacity.

360 In light of the preceding study, our findings for the realistic simula361 tion parameters in Table I may be summarized as follows.

363 1) The performance of the suboptimal scheme as a percentage of 364 the throughput achieved by the optimal scheme reaches its max365 imum when the two phases of the successive relaying protocol 366 have equal duration.

367 2) The optimal and suboptimal schemes are capable of achieving 368 up to $92 \%$ and $88 \%$ of the benchmark scheme's throughput [3] 369 for sufficiently high energy and data buffer capacities.

370 3) The suboptimal scheme's throughput is consistently about $90 \%$ 371 of that of the optimal scheme.

372 4) For asymmetric data (or energy) buffer sizes, the attainable 373 throughput depends on the total (i.e., collective) data (or energy) 374 buffer capacity available in the network and not only on the 375 smallest data buffer.

\section{CONCLUSION}

377 In this paper, we have considered the throughput optimization of an $378 \mathrm{EH}$-assisted two-hop network using a buffer-aided successive relaying 379 protocol. Under the assumption of known energy arrivals, we defined 380 the related nonconvex optimization problem and proposed both opti$381 \mathrm{mal}$ and suboptimal schemes to maximize the data delivered to the $D N$ 382 by the deadline. Then, using the interior-point method, an efficient 383 solution was found for both schemes. Finally, our results justify that 384 both our optimal and suboptimal schemes are capable of performing 385 close to the benchmark system [3]. Furthermore, the less-complex 386 suboptimal scheme is capable of approaching the performance of our 387 optimal scheme at the expense of a slight performance degradation, provided that the $\mathrm{EH}$ nodes are equipped with sufficiently large buffers 388 for both energy and data storage. Our future work may consider 389 $\mathrm{EH}$-aided adaptive transceiver schemes.

\section{APPENDIX A}

PROOF OF LEMMA 1

This proof is an extension of that derived for the point-to-point case 393 in [5] to the two-hop scenario defined in this paper. Let us assume that 394 the transmitter nodes $\left(S N, R N_{1}, R N_{2}\right)$ change their transmission rate 395 between two $\mathrm{EH}$ instances $t_{i}$ and $t_{i+1}$. Let us furthermore denote the 396 rates by $r_{M, n}$ and $r_{M, n+1}$ and the instant when the rate changes by 397 $t_{i}^{\prime}$, where we have $M \in\{S I, R 2\}$ in Phase I and $M \in\{S I I, R 1\} 398$ in Phase II of the successive relaying protocol. Correspondingly, the 399 duration of each phase can be written as $L_{I, n}, L_{I, n+1}, L_{I I, n}$, and 400 $L_{I I, n+1}$. Let us now consider the duration $\left[t_{i}, t_{i+1}\right)$. The total energy 401 consumed in this duration at $S N$ is $p_{S I, n} L_{I, n}+p_{S I I, n} L_{I I, n}+402$ $p_{S I, n+1} L_{I, n+1}+p_{S I I, n+1} L_{I I, n+1}$. Similarly, the total energy con- 403 sumed at $R N_{1}$ is $p_{R 1, n} L_{I I, n}+p_{R 1, n+1} L_{I I, n+1}$ and that at $R N_{2}$ is 404 $p_{R 2, n} L_{I, n}+p_{R 2, n+1} L_{I, n+1}$. Let us now consider $S N$ in more detail 405 and define

$$
\begin{aligned}
p_{S I}^{\prime} & =\frac{p_{S I, n} L_{I, n}+p_{S I, n+1} L_{I, n+1}}{t_{i+1}-t_{i}} \\
p_{S I I}^{\prime} & =\frac{p_{S I I, n} L_{I I, n}+p_{S I I, n+1} L_{I I, n+1}}{t_{i+1}-t_{i}} \\
r_{S I}^{\prime} & =r\left[p_{S I}^{\prime}\right]=r\left[\frac{p_{S I, n} L_{I, n}+p_{S I, n+1} L_{I, n+1}}{t_{i+1}-t_{i}}\right] \\
r_{S I I}^{\prime} & =r\left[p_{S I I}^{\prime}\right]=r\left[\frac{p_{S I I, n} L_{I I, n}+p_{S I I, n+1} L_{I I, n+1}}{t_{i+1}-t_{i}}\right] .
\end{aligned}
$$

Let us now use these $r_{S I}^{\prime}$ and $r_{S I I}^{\prime}$ as the new transmission rates for 407 Phases I and II at $S N$ over $\left[t_{i}, t_{i+1}\right)$ and keep the rest of the rates 408 the same as in the original policy. It is easy to observe that the new 409 transmission policy is feasible, since all the energy constraints are 410 satisfied under this policy. On the other hand, we can write the total 411 number of packets that are departed from $S N$ in both of the phases 412 over this duration under this new policy as

$$
\begin{aligned}
\left(r_{S I}^{\prime}+r_{S I I}^{\prime}\right)\left(t_{i+1}-t_{i}\right)=\left(r\left[p_{S I}^{\prime}\right]+r\left[p_{S I I}^{\prime}\right]\right)\left(t_{i+1}-t_{i}\right) \\
=\left(r\left[\frac{p_{S I, n} L_{I, n}+p_{S I, n+1} L_{I, n+1}}{t_{i+1}-t_{i}}\right]\right)\left(t_{i+1}-t_{i}\right) \\
\quad+\left(r\left[\frac{p_{S I I, n} L_{I I, n}+p_{S I I, n+1} L_{I I, n+1}}{t_{i+1}-t_{i}}\right]\right)\left(t_{i+1}-t_{i}\right) \\
\geq \\
\quad\left(r\left[p_{S I, n}\right] L_{I, n}+r\left[p_{S I, n+1}\right] L_{I, n+1}\right) \\
\quad+\left(r\left[p_{S I I, n}\right] L_{I I, n}+r\left[p_{S I I, n+1}\right] L_{I I, n+1}\right) \\
=r_{S I, n} L_{I, n}+r_{S I, n+1} L_{I, n+1}+r_{S I I, n} L_{I I, n} \\
\quad+r_{S I I, n+1} L_{I I, n+1}
\end{aligned}
$$

where the inequality in (5b) follows from (1) in Section II, which is 414 a concave function of the transmission power $p$. Therefore, the total 415 number of packets transmitted by $S N$ in this duration under the new 416 policy is higher than those that are departed under the original policy. 417 Similarly, we can prove that the RNs under this new policy will send 418 more data to $D N$. If we keep all the rates constant, the transmissions 419 will deliver larger amounts of data to $D N$ by the deadline. This 420 contradicts the optimality of the original transmission policy. 
424 The proof derived for the two-relay case extends the single-relay 425 case of [14]. In the case of two parallel relays, we consider a feasible 426 transmission policy where one of the relays (e.g., $R N_{1}$ ) is not always 427 on, i.e., it is not transmitting or receiving data all the time. Now, if 428 we have an idle time interval right at the beginning of Phase I, we can 429 extend the epoch of $S N$ in Phase II, ensuring that there is no idle time. 430 Note that this strategy continues to satisfy all the causality and storage 431 constraints. On the other hand, if an idle time duration occurs at the 432 beginning of Phase II, we can delay the epoch of relay $R N_{1}$ without 433 violating the feasibility of our policy, because it can store more energy 434 in the meantime, and the previous argument can be used to extend the 435 epoch of $R N_{2}$ during Phase I to avoid any idle time. Similarly, we 436 can consider the scenario when $R N_{2}$ is not always on. Therefore, we 437 remove the idle times by increasing the transmission duration of one of 438 the nodes ( $S N$ or RNs) while keeping the total amount of transmitted 439 data the same. Since the rate-power relation of (1) is concave, the new 440 policy conveys the same amount of data to $D N$ while consuming less 441 energy. Hence, it is feasible. Moreover, using this proof, we can say 442 that there exists an optimal policy, where $S N$ and $D N$ are always on 443 for the twin-relay system relying on a successive relaying protocol.

\section{4 \\ 445 \\ INTERIOR-POINT OPTIMIZATION METHOD}

446 The relevant optimization techniques include IPOPT, LOQO, and 447 KNITRO [15]. The IPOPT method is more efficient than the other 448 two techniques, because it relies on tighter termination bounds and 449 utilizes comparable CPU time to evaluate a higher number of objective 450 function values and iterations [15]. The IPOPT method involves the 451 primal-dual interior-point algorithm with the aid of a so-called filter 452 line-search method invoked for nonlinear programming [15], [16], 453 which improves its robustness over that of LOQO and KNITRO. In the 454 primal-dual interior-point method, both primal and dual variables are 455 updated, whereas primal and dual iterates do not have to be feasible. 456 The search direction in this method is obtained using Newton's method 457 applied to the modified Karush-Kuhn-Tucker equations. However, the 458 basic idea behind the filter line-search algorithm involves considering 459 a trial point during the backtracking line search, where this trial point 460 is considered to be acceptable if it leads to sufficient progress toward 461 achieving the optimization goal. This algorithm maintains a "filter," 462 which is a set of values that both the objective function and the 463 constraint violation functions are prohibited from returning. For a trial 464 point to be successful, the values of the objective function and the constraint violation functions evaluated at that trial point should not 465 be a member of the filter. This filter is updated at every iteration to 466 ensure that the algorithm does not cycle in the neighborhood of the 467 previous iterate [15].

\section{REFERENCES}

[1] B. Medepally and N. Mehta, "Voluntary energy harvesting relays 470 and selection in cooperative wireless networks," IEEE Trans. Wireless 471 Commun., vol. 9, no. 11, pp. 3543-3553, Nov. 2010.

[2] I. Ahmed, A. Ikhlef, R. Schober, and R. Mallik, "Power allocation for 473 conventional and buffer-aided link adaptive relaying systems with en- 474 ergy harvesting nodes," IEEE Trans. Wireless Commun., vol. 13, no. 3, 475 pp. 1182-1195, Mar. 2014.

[3] O. Orhan and E. Erkip, "Throughput maximization for energy harvesting 477 two-hop networks," in Proc. IEEE ISIT, Jul. 2013, pp. 1596-1600. 478

[4] C. Murthy and N. Mehta, "Tutorial on energy harvesting wireless com- 479 munication systems," in Proc. Nat. Conf. Commun., Kanpur, India, 480 Feb. 2014, pp. 1596-1600.

[5] J. Yang and S. Ulukus, "Optimal packet scheduling in an energy har- 482 vesting communication system," IEEE Trans. Commun., vol. 60, no. 1, 483 pp. 220-230, Jan. 2012.

[6] K. Tutuncuoglu and A. Yener, "Optimum transmission policies for battery 485 limited energy harvesting nodes," IEEE Trans. Wireless Commun., vol. 11, 486 no. 3, pp. 1180-1189, Mar. 2012.

[7] P. He, L. Zhao, S. Zhou, and Z. Niu, "Recursive waterfilling for wireless 488 links with energy harvesting transmitters," IEEE Trans. Veh. Technol., 489 vol. 63, no. 3, pp. 1232-1241, Mar. 2014.

490

[8] J. Yang, O. Ozel, and S. Ulukus, "Broadcasting with an energy harvesting 491 rechargeable transmitter," IEEE Trans. Wireless Commun., vol. 11, no. 2, 492 pp. 571-583, Feb. 2012.

[9] C.-C. Kuan, G.-Y. Lin, H.-Y. Wei, and R. Vannithamby, "Reli- 494 able multicast and broadcast mechanisms for energy-harvesting de- 495 vices," IEEE Trans. Veh. Technol., vol. 63, no. 4, pp. 1813-1826, 496 May 2014.

[10] Z. Fang, T. Song, and T. Li, "Energy harvesting for two-way OFDM 498 communications under hostile jamming," IEEE Signal Process. Lett., 499 vol. 22, no. 4, pp. 413-416, Apr. 2015.

[11] K. Tutuncuoglu, B. Varan, and A. Yener, "Optimum transmission poli- 501 cies for energy harvesting two-way relay channels," in Proc. IEEE ICC, 502 Jun. 2013, pp. 586-590.

[12] I. Ahmed, A. Ikhlef, D. Ng, and R. Schober, "Optimal resource allocation 504 for energy harvesting two-way relay systems with channel uncertainty," 505 Proc. IEEE GlobalSIP, Dec. 2013, pp. 345-348.

506

[13] N. Roseveare and B. Natarajan, "An alternative perspective on utility max- 507 imization in energy-harvesting wireless sensor networks," IEEE Trans. 508 Veh. Technol., vol. 63, no. 1, pp. 344-356, Jan. 2014.

[14] O. Orhan and E. Erkip, "Optimal transmission policies for energy har- 510 vesting two-hop networks," in Proc. 46th Annu. Conf. CISS, Mar. 2012, 511 pp. 1-6.

512

[15] A. Wachter and L. T. Biegler, "On the implementation of an interior-point 513 filter line-search algorithm for large-scale nonlinear programming," Math. 514 Programm., vol. 106, no. 1, pp. 25-57, Mar. 2006.

[16] S. Boyd and L. Vandenberghe, Convex Optimization. New York, NY, 516 USA: Cambridge Univ. Press, 2004. 


\section{AUTHOR QUERIES}

\section{AUTHOR PLEASE ANSWER ALL QUERIES}

$\mathrm{AQ} 1=$ Please provide keywords.

AQ2 = Table 1 was cited and captured as text. Please check.

END OF ALL QUERIES 


\section{Correspondence}

\section{Throughput Maximization for a Buffer-Aided Successive 2 Relaying Network Employing Energy Harvesting}

\author{
3 Shruti Gupta, Rong Zhang, and Lajos Hanzo
}

4 Abstract-Energy harvesting (EH)-assisted nodes are capable of sig5 nificantly prolonging the lifetime of future wireless networks, provided 6 that they rely on appropriate transmission policies, which accommo7 date the associated stochastic energy arrival. In this paper, a successive8 relaying-based network using rechargeable source and relay nodes having 9 limited buffers for both their energy and data storage is considered. The 10 maximization of the network throughput with noncausal knowledge of en11 ergy arrivals by the deadline $\boldsymbol{T}$ is formulated as a nonconvex optimization 12 problem, and it is solved using the interior-point optimization (IPOPT) 13 method. The performance of the low-complexity suboptimal scheme was 14 found to reach its maximum when the two phases of the successive relaying 15 protocol have equal duration. The optimal and suboptimal schemes are 16 capable of achieving up to $92 \%$ and $88 \%$ of the throughput performance of 17 the benchmark scheme. The suboptimal scheme's throughput performance 18 is consistently about $90 \%$ of that of the optimal scheme. For asymmetric 19 data (or energy) buffer sizes, it was found that the throughput performance 20 depends on the total (i.e., collective) data (or energy) buffer capacity 21 available in the network and not just on the smallest data buffer.

22 Index Terms-Author, please supply index terms/keywords for your 23 paper. To download the IEEE Taxonomy go to http://www.ieee.org/ 24 documents/taxonomy_v101.pdf.

\section{INTRODUCTION}

26 Cooperative communication is capable of attaining significant 27 throughput and reliability improvements, where the source node $(S N)$ 28 and cooperating relay nodes $(R N)$ expend their energy while process29 ing and transmitting the signal to the destination node $(D N)$. The 30 nodes are typically powered through precharged batteries, but once 31 these batteries are drained, the nodes become dysfunctional [1], [2]. An 32 emerging solution to this vexed problem is the use of energy harvesting 33 (EH) [1]-[3], which has to be capable of accommodating the random 34 arrivals of energy and its storage at the nodes [4].

35 Hence, EH communication systems have been studied under dif36 ferent network models. In [5]-[7], a single-user EH system was char37 acterized, where beneficial power-allocation strategies were designed 38 under the corresponding EH constraints. This was further extended to 39 the design of an EH-aided broadcast channel in [8] and [9] and to 40 two-way orthogonal frequency-division multiplexing communications 41 [10]. In [8], Yang et al. defined the cutoff power levels for each 42 user to allocate the optimal power to them, whereas in [9], Kuan 43 et al. analyzed the tradeoff between the achievable reliability and 44 throughput for broadcast transmissions relying on erasure codes for $45 \mathrm{EH}$ sensors. In [10], the receiver is designed both for simultaneously

Manuscript received January 28, 2015; revised June 3, 2015 and July 30, 2015; accepted September 8, 2015. This work was supported by the European Research Council under the Advanced Fellow Grant. The review of this paper was coordinated by Dr. L. Zhao.

The authors are with the School of Electronics and Computer Science, University of Southampton, Southampton SO17 1BJ, U.K. (e-mail: sg7g12@ ecs.soton.ac.uk; rz@ecs.soton.ac.uk; 1h@ecs.soton.ac.uk).

Color versions of one or more of the figures in this paper are available online at http://ieeexplore.ieee.org.

Digital Object Identifier 10.1109/TVT.2015.2477808

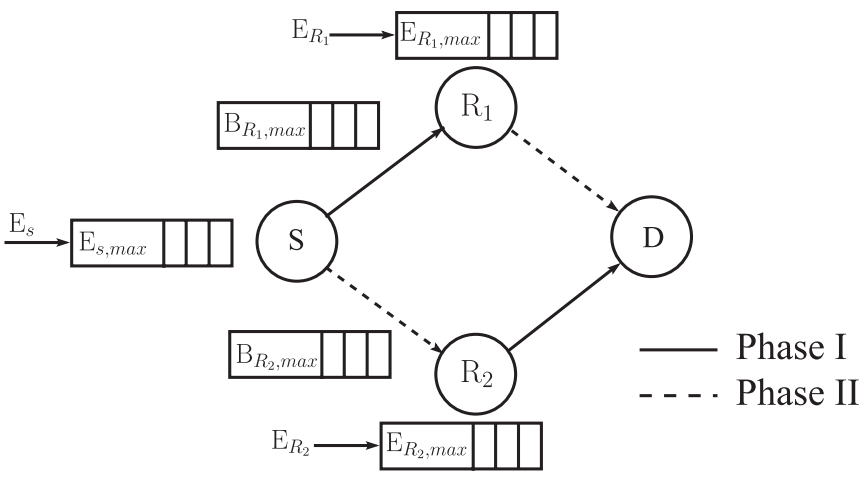

Fig. 1. Successive relaying network where EH nodes are equipped with finite buffer for both energy and data storage.

processing information and for harvesting energy from the received 46 desired signal, as well as jamming interference a through power 47 splitter. In recent years, cooperative networks have also been studied 48 in the context of EH at the RNs and/or the $S N$ [1]-[3], [11]-[13]. 49 Specifically, in [1], Medepally and Mehta investigated the benefits 50 of relay selection relying on multiple EH amplify-and-forward RNs, 51 whenever they have sufficient energy for transmission. By contrast, 52 in [2], information-buffer-aided link activation was used, which was 53 controlled both by the quality of the links and by the amount of 54 energy buffered at these nodes. Two-hop networks relying either on a 55 single or on a pair of parallel RNs using a successive relaying protocol 56 were investigated to quantify the benefits of both multiple relays and 57 of $\mathrm{EH}$ on the average throughput of the system in [3]. In [11], the 58 authors derived the optimal achievable rates for an EH system in the 59 context of two-way relaying employing different relaying strategies. 60 Furthermore, a similar two-way EH relay system employing time- 61 division broadcasting and multiple access broadcasting, which was 62 subjected to channel state uncertainty, was considered in the context 63 of joint energy and transmit time allocation in [12]. Utilizing the struc- 64 ture of a specific problem and the generalized optimality principle, 65 in [13], a new algorithm for constrained utility maximization problems 66 encountered in a cooperative network of wireless sensor nodes is 67 formulated.

Against this background, we consider a successive relaying model, 69 which is capable of mimicking a full-duplex (FD) $R N$, despite relying 70 on a pair of half-duplex (HD) RNs, which are activated alternately in 71 their transmitter and receiver modes to create a virtual FD relay. This 72 HD regime reduces the complexity of the FD system, since the FD 73 $R N$ would require high-complexity interference cancellation at the 74 receiver. In contrast to [3], our model relies on the realistic constraint 75 that $\mathrm{EH}$ nodes $\left(S N, R N_{1}, R N_{2}\right)$ have a finite energy storage capacity 76 and that the RNs also have limited data buffers for storing the source 77 data. We first formulate an optimization problem for the throughput 78 maximization of our successive-relaying-aided network in Fig. 1 hav- 79 ing finite buffers, as well as relying on the idealized noncausal 80 knowledge of the energy arrivals at all EH nodes. Then, using the 81 interior-point optimization method (IPOPT), the optimization problem 82 is solved for both optimal and suboptimal schemes, and finally, we 83 quantify the effect of buffer sizes on the throughput of the network 84 based on both schemes. While proof-of-concept studies are indeed 85 
86 valuable, the ultimate purpose of most engineering studies is to attempt 87 a real-world implementation of the proposed techniques. Through this 88 study, we aimed to take the valuable proposals in [3] a step closer to 89 its real-world deployment. Explicitly, the novelty of this contribution 90 is given as follows: 1) We define a practical successive relaying model 91 constrained by both limited energy and data storage buffers at the EH 92 nodes, which dispenses with the idealized simplifying assumption of 93 having infinite buffers [3]; 2) we formulate the optimal transmission 94 policy; and 3) we propose a suboptimal transmission scheme capable 95 of approaching the performance of its optimal counterpart at signif96 icantly reduced complexity, which is achieved at the expense of a 97 marginally degraded performance. In our study, we also consider the 98 scenario of asymmetric fading, energy, and data buffers. This paper is 99 organized as follows. In Section II, our system model is presented, 100 which is followed by the formulation of our optimization problem 101 in Section III. Our results are discussed in Section IV, whereas our 102 conclusions are offered in Section V.

\section{SySTEM MODEL}

104 We consider the successive relaying technique of [3] having two 105 phases, where the RNs assist the SN's transmission to the $D N$, as 106 shown in Fig. 1. In Phase I in Fig. 1 , the $S N$ transmits to $R N_{1}$, 107 whereas $R N_{2}$ simultaneously transmits to the $D N$. By contrast, in 108 Phase II in Fig. 1, $S N$ and $R N_{1}$ transmit simultaneously both to $109 R N_{2}$ and to $D N$, respectively. Thus, the $S N$ is always transmitting, 110 whereas the $D N$ is always receiving during the process. It is assumed 111 that there is no direct link between $S N-D N$ and $R N_{1}-R N_{2}$, as well 112 as that these are decode-and-forward (DF) HD RNs that are located 113 sufficiently far apart from each other to avoid any interference. We 114 assume that $S N, R N_{1}$, and $R N_{2}$ harvest energy from the environment 115 and have finite energy buffers that can store a maximum of $E_{S, \max }$, $116 E_{R 1, \max }$, and $E_{R 2 \text {, max }}$ units, respectively, whereas $R N_{1}$ and $R N_{2}$ 117 are also equipped with data buffers of $B_{R 1 \text {,max }}$ and $B_{R 2 \text {,max }}$ packets, 118 respectively. For ease of exposition, we merge the energy arrival events 119 at all the $\mathrm{EH}$ nodes into a single time series $\left(t_{0}, t_{1}, \ldots, t_{K}\right)$ by consid120 ering zero amount of energy arrivals at the nodes that do not harvest 121 energy at some instant $t_{k}$. More explicitly, the $\mathrm{EH}$ processes at the $\mathrm{EH}$ 122 nodes are independent of each other. In other words, the energy arrival 123 instances of a node may be different from those of the other nodes. 124 For example, assume that an energy arrival occurred at node $R N_{1}$ at 125 some instant $t_{k}$, whereas there was no energy arrival at the other nodes 126 ( $S$ and $R N_{2}$ ) at the time instant $t_{k}$. In our mathematical analysis, 127 we assumed that at time instant $t_{k}$, nodes $S$ and $R N_{2}$ harvested 128 zero amount of energy. We set $t_{0}=0$ and $t_{K}=T$. We represent the 129 amount of energy harvested at $S N, R N_{1}$, and $R N_{2}$ at time instant $t_{k}$ 130 as $E_{S, k}, E_{R 1, k}$, and $E_{R 2, k}$ units, respectively, for $k=0,1, \ldots K-1$. 131 The time interval between the two consecutive energy arrivals is 132 termed as an epoch, whose length is defined as $\tau_{k}=t_{k}-t_{k-1}$. The 133 complex-valued channel gains are considered to be constant through134 out the communication process preceding the deadline. The channel 135 gain between the nodes $L$ and $M$ is denoted by $H_{L M}$, where we have $136 L \in\left\{S N, R_{1} N, R_{2} N\right\}$ and $M \in\left\{R N_{1}, R N_{2}, D N\right\}$.

137 We consider the throughput maximization problem under the ide138 alized simplifying assumption of having prior knowledge about the 139 energy arrivals at all the EH nodes before the commencement of 140 the communication process. We assume that the energy expended at 141 the nodes is only the transmission energy and that perfect "capacity142 achieving" codes are used, which facilitate operation exactly at the 143 Shannon capacity, thus determining the rate versus power relationship 144 of a given link, which is given by

$$
r[p(t)]=\log _{2}[1+H p(t)]
$$

where $H$ is the channel gain of the link, and $p(t)$ is the transmission 145 power of the node at time $t$. As a result of energy arrivals over time and 146 as a benefit of the energy storage capacity at the nodes, any feasible 147 transmission policy should satisfy following constraints.

1) Energy causality constraint: The total energy expended by a 150 node during its transmission session should not exceed the total 151 energy harvested by that node until that time.

2) Energy overflow constraint: The energy exceeding the storage 153 capacity of the energy buffer at the node is lost owing to 154 overflow.

3) Data causality constraint: The total data transmitted by a node 156 during the process should not exceed the total data received by 157 that node until that time.

4) Data overflow constraint: The amount of data exceeding the 159 storage capacity of data buffer is lost due to overflow.

\section{Problem Formulation}

Here, we first stipulate some properties of the optimal transmission 162 policy in the following two lemmas, which will be used to formulate 163 the throughput maximization problem for the system in Fig. 1. The 164 proof of these lemmas is provided in Appendixes A and B. 165

Lemma 1: The transmission rate/power of a node is constant be- 166 tween two consecutive energy arrivals but potentially changes when 167 new energy arrives at the node [3].

Lemma 2: The feasible transmission policy ensures that the relays 169 are always on without decreasing the throughput of the system [3]. 170

Based on Lemmas 1 and 2, we can characterize the optimal policy in 171 the following way. There is a constant transmission rate for the pair of 172 nodes between consecutive energy arrivals according to the optimal 173 policy, as formulated in Lemma 1. Therefore, we assume that the 174 transmission power of $S N$ during Phases I and II in Fig. 1 in an epoch 175 is constant and given by $p_{S I, k}$ and $p_{S I I, k}$, respectively. Similarly, 176 the transmission power of $R N_{1}$ and $R N_{2}$ is denoted by $p_{R_{1}, k}$ and 177 $p_{R_{2}, k}$, respectively. Lemma 2 implies that we restrict our attention 178 to the specific transmission policies, where both $R N_{1}$ and $R N_{2}$ are 179 always on for the sake of defining a feasible transmission policy. Thus, 180 we assume that the total transmission time between $S N-R N_{1}$ and 181 $R N_{2}-D N$ is the same and denote this duration of Phase I between 182 the time instants $t_{k-1}$ and $t_{k}$ by $L_{I, k}$. Similarly, we assume the same 183 transmission time between $S N-R N_{2}$ and $R N_{1}-D N$ in Phase II, 184 which is denoted by $L_{I I, k}, k=1,2, \ldots, K$. Finally, we identify 185 the optimal transmission policy that defines which particular node 186 transmits and when, along with the specific power allocation of each 187 node. We then define a suboptimal scheme, where the duration of each 188 phase of successive relaying is fixed to a particular ratio.

\section{A. Optimal Transmission Policy}

Let us now define the optimization problem of maximizing the 191 system throughput by the deadline $T$. Since $R N_{2}$ initially has no data 192 in Phase I in Fig. 1, it is assumed without loss of generality that it 193 starts transmission by delivering $\epsilon>0$ amount of dummy information 194 to $D N$, where $\epsilon$ is sufficiently small to be ignored for our throughput 195 optimization problem. Upon scheduling the two phases in succession, 196 it is ensured that there is no further throughput loss for the system. 197 In other words, at the beginning of transmission, $R N_{2}$ possesses no 198 data from $S$ that can be transmitted to $D N$; hence, it commences its 199 transmission with $\epsilon$ dummy packets. However, subsequently, the trans- 200 mission phases occur in immediate succession without any interval. 201 This ensures that there is no need to send dummy packets, and thus, 202 no further loss of system throughput is imposed. Similar assumptions 203 were also made in [3]. We first define the throughput of the nodes 204 
205 in different phases based on the rate versus power relationship (1) 206 mentioned in Section II as

$$
\begin{aligned}
\alpha_{R 1, k} & =L_{I I, k} \log _{2}\left(1+H_{R 1 D} p_{R 1, k}\right) \\
\alpha_{R 2, k} & =L_{I, k} \log _{2}\left(1+H_{R 2 D} p_{R 2, k}\right) \\
\alpha_{S I, k} & =L_{I, k} \log _{2}\left(1+H_{S R 1} p_{S I, k}\right) \\
\alpha_{S I I, k} & =L_{I I, k} \log _{2}\left(1+H_{S R 2} p_{S I I, k}\right) .
\end{aligned}
$$

207 Now, the optimization problem is defined over $L_{I, k}, L_{I I, k}, \alpha_{S I, k}$, $208 \alpha_{S I I, k}, \alpha_{R 1, k}$, and $\alpha_{R 2, k},(3 \mathrm{a})-(3 \mathrm{~m})$, shown at the bottom of the page. 209 Note that when (3h)-(3i) are evaluated at $k=K$, the total amount 210 of data delivered to $D N$ is equal to the amount of data transferred by $R N_{1}$ and $R N_{2}$; hence, the throughput maximization problem 211 corresponds to the maximization of the amount of data transmitted 212 by both the RNs, as formulated in (3a). The problem in (3) is a non- 213 convex optimization problem, owing to the nonconvex energy storage 214 constraints defined in (3e)-(3g), which can be efficiently solved using 215 the IPOPT method, as given in Appendix C.

B. Suboptimal (Alternate) Transmission Policy

In this scheme, we set the duration of Phase I in Fig. 1 to be equal 218 to $\eta \%$; of the length of an epoch, i.e., we have

$$
L_{I, k}=\frac{\eta}{100} \tau_{k}, \quad L_{I I, k}=\tau_{k}-\frac{\eta}{100} \tau_{k} .
$$

$\operatorname{maximize} \quad \sum_{k=1}^{K} \alpha_{R 1, k}+\alpha_{R 2, k}$

subject to

Energy causality constraints (constraint 1 in Section II) at $S N, R N_{1}$, and $R N_{2}$ :

$$
\begin{aligned}
& \sum_{j=1}^{k} \frac{L_{I, j}}{H_{S R 1}}\left(2\left(\frac{\alpha_{S I, j}}{L_{I, j}}\right)-1\right)+\frac{L_{I I, j}}{H_{S R 2}}\left(2^{\left(\frac{\alpha_{S I I, j}}{L_{I I, j}}\right)}-1\right) \leq \sum_{j=0}^{k-1} E_{S, j} \quad \forall k \\
& \sum_{j=1}^{k} \frac{L_{I I, j}}{H_{R 1 D}}\left(2^{\left(\frac{\alpha_{R I, j}}{L_{I I, j}}\right)}-1\right) \leq \sum_{j=0}^{k-1} E_{R 1, j} \quad \forall k \\
& \sum_{j=1}^{k} \frac{L_{I, j}}{H_{R 2 D}}\left(2^{\left(\frac{\alpha_{R 2, j}}{L_{I, j}}\right)}-1\right) \leq \sum_{j=0}^{k-1} E_{R 2, j} \quad \forall k .
\end{aligned}
$$

Energy overflow constraints (constraint 2 in Section II) at $S N, R N_{1}$, and $R N_{2}$ :

$$
\begin{aligned}
& \sum_{j=0}^{k} E_{S, j}-\sum_{j=1}^{k} \frac{L_{I, j}}{H_{S R 1}}\left(2\left(\frac{\alpha_{S I, j}}{L_{I, j}}\right)-1\right)+\frac{L_{I I, j}}{H_{S R 2}}\left(2^{\left(\frac{\alpha_{S I I, j}}{L_{I I, j}}\right)}-1\right) \leq E_{S, \max } \quad \forall k \\
& \sum_{j=0}^{k} E_{R 1, j}-\sum_{j=1}^{k} \frac{L_{I I, j}}{H_{R 1 D}}\left(2\left(\frac{\alpha_{R 1, j}}{L_{I I, j}}\right)-1\right) \leq E_{R 1, \max } \quad \forall k \\
& \sum_{j=0}^{k} E_{R 2, j}-\sum_{j=1}^{k} \frac{L_{I, j}}{H_{R 2 D}}\left(2\left(\frac{\alpha_{R 2, j}}{L_{I, j}}\right)_{i}-1\right) \leq E_{R 2, \max } \quad \forall k .
\end{aligned}
$$

Data causality constraints (constraint 3 in Section II) at $R N_{1}$ and $R N_{2}$ :

$$
\begin{array}{ll}
\sum_{j=1}^{k} \alpha_{R 1, j} \leq \sum_{j=1}^{k} \alpha_{S I, j} & \forall k \\
\sum_{j=1}^{k} \alpha_{R 2, j} \leq \sum_{j=1}^{k} \alpha_{S I I, j} & \forall k .
\end{array}
$$

Data overflow constraints (constraint 4 in Section II) at $R N_{1}$ and $R N_{2}$ :

$$
\begin{array}{ll}
\sum_{j=1}^{k} \alpha_{S I, j}-\sum_{j=1}^{k-1} \alpha_{R 1, j} \leq B_{R 1, \max } & \forall k \\
\sum_{j=1}^{k} \alpha_{S I I, j}-\sum_{j=1}^{k-1} \alpha_{R 2, j} \leq B_{R 2, \max } & \forall k .
\end{array}
$$

Half duplex constraint due to the HD relays $R N_{1}$ and $R N_{2}$ :

$L_{I, k}+L_{I I, k} \leq \tau_{k} \quad \forall k$.

$$
\forall k \text {. }
$$

Feasibility constraints at $S N, R N_{1}$ and $R N_{2}$ :

$\alpha_{S I, k} \geq 0, \quad \alpha_{S I I, k} \geq 0, \quad \alpha_{R 1, k} \geq 0 ; \alpha_{R 2, k} \geq 0, \quad L_{I, k} \geq 0, \quad L_{I I, k} \geq 0 \quad \forall k$ 


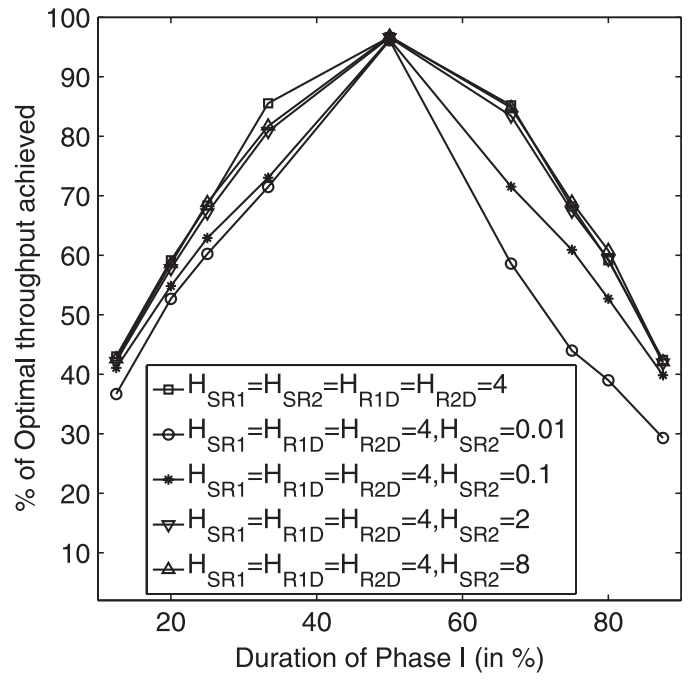

Fig. 2. Relation between percentage of optimal throughput achieved for varying duration of Phase I occurring in an EH epoch with sufficient energy and data buffer sizes (5 and 2, respectively) for different settings of channel gains.

220 Using (4), the optimization problem is relaxed for this suboptimal 221 scheme and can be reformulated by omitting (31) from (3). This is 222 again a nonconvex optimization problem; hence, it may be solved 223 using the IPOPT method. This scheme is termed suboptimal, since 224 the duration of the phases has been deliberately fixed for the sake of 225 reducing the complexity ${ }^{1}$ of the optimization problem.

\section{Performance Results}

227 Here, we evaluate the performance of the proposed buffer-aided 228 successive relaying system relying on offline power allocation in 229 terms of the optimal throughput achieved by the deadline of $T=$ $23010 \mathrm{~s}$. We assume that the EH process of both the $S N$ and the RNs 231 independently takes values from $\left[0, E_{\max }=5\right]$ units, where the energy 232 is uniformly distributed under an exponential inter-arrival time at 233 a rate of $\lambda_{e}=5$ units/s. The deterministic channel gains are set to 234 the values $H_{S R 1}=H_{S R 2}=H_{R 1 D}=H_{R 2 D}=4$, except otherwise 235 mentioned. Our results quantify the throughput of the system as a 236 function of both data and energy buffer capacity for both optimal 237 and suboptimal schemes that are benchmarked against the infinite238 storage-based optimal scheme defined in [3]. Our benchmark scheme 239 of [3] is insensitive to the buffer sizes, since it considers infinite 240 storage capacities at all the EH nodes for both energy and data, thereby 241 providing an upper bound to our proposed system.

242 The percentage duration of Phases I and II in Fig. 1 is not fixed 243 for the optimal scheme, whereas they have been fixed to a specific 244 ratio for the suboptimal scheme for the sake of complexity reduction. 245 Hence, our first goal was to identify the specific ratio of the durations 246 of Phases I and II that would maximize the throughput of the sub247 optimal scheme. Fig. 2 shows the specific percentage of the optimal 248 throughput, which was actually achieved by varying the proportion of 249 the Phase I duration $\left(L_{I}\right)$ in each of the EH epochs, along with the 250 symmetric $\left(H_{S R 1}=H_{S R 2}=H_{R 1 D}=H_{R 2 D}=4\right)$ and asymmetric 251 settings of the channel fading gain for $S N-R N_{2}$. The performance 252 of the suboptimal scheme peaks when the durations of both phases 253 are equal. For the other scenarios, the throughput is lower, because the 254 amount of data transmitted between $S N$ and $D N$ is limited by the 255 shorter phase. It is shown in Fig. 2 that, as the duration of the shorter 256 phase increases, the throughput also increases. It is interesting to note

${ }^{1}$ The complexity analysis of both schemes is beyond the scope of this paper.

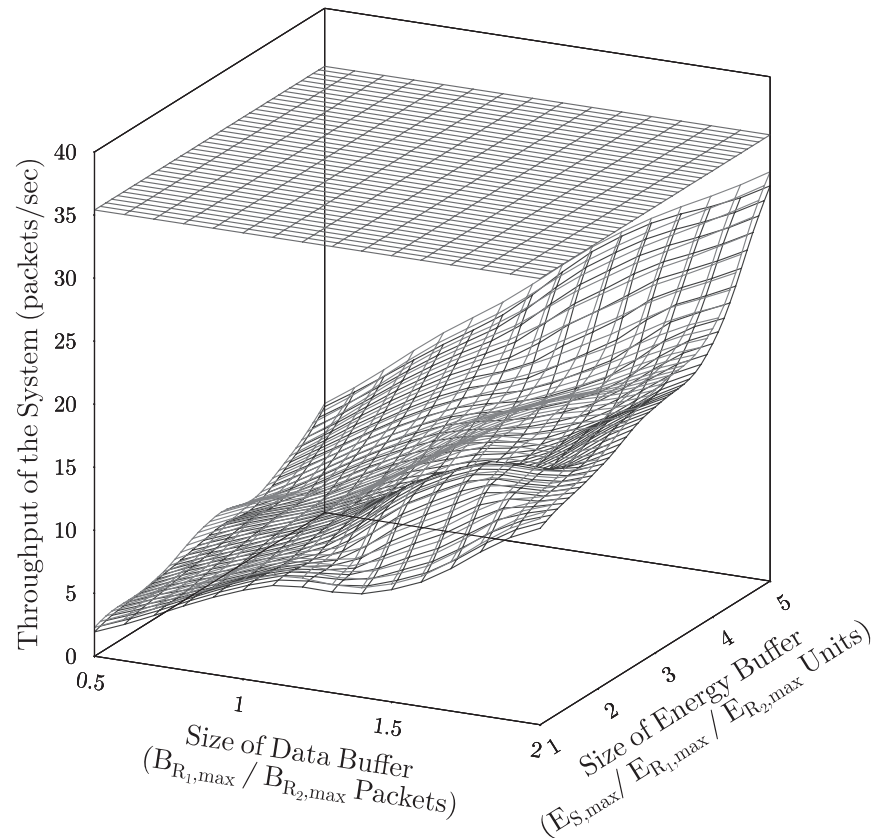

Fig. 3. Impact of the energy and data buffer sizes at all the EH nodes on the throughput of the system by the deadline $T$. The constant green surface represents the throughput of the benchmark scheme [3], whereas the pink and blue surfaces depict our optimal and suboptimal transmission policies, respectively.

that in the scenarios of very low channel gain, i.e., for $H_{S R 2}=0.01257$ and $H_{S R 2}=0.1$, there exists asymmetry in the throughput achieved 258 by the system. The reason behind this trend is that when the duration 259 of Phase I is higher than that of Phase II, the channel gain of path 260 $S N-R N_{2}$ limits the amount of data that can be otherwise transmitted 261 to $R N_{2}$. As shown in Fig. 2, when the duration of Phase I is $50 \%$ of 262 the EH epoch, the suboptimal scheme achieves approximately $97 \%$ of 263 the optimal scheme's throughput. Hence, in the following discussions, 264 we consider a suboptimal scheme, where the duration of each phase is 265 $50 \%$ of the epoch duration.

The 3-D characterization of the system in Fig. 1 is provided in 267 Fig. 3. Specifically, Fig. 3 shows the overall throughput of the system 268 as a function of the size of both energy and data buffers at the EH 269 nodes. It can be clearly observed that, with the increase in the size of 270 buffers at the EH nodes, the throughput of our proposed schemes 271 improve owing to increased availability of energy and data storage 272 capacity at the EH nodes, supporting a larger amount of data trans- 273 mission to $D N$. However, the throughput of the benchmark scheme 274 [3] is constant, i.e., independent of the buffer sizes, as it relies on the 275 idealized settings where EH nodes possess infinite energy and data 276 storage capacity. Moreover, our optimal scheme performs only mar- 277 ginally better than our less complex suboptimal scheme, because the 278 duration of each phase is fixed in the suboptimal scheme. This would, 279 in turn, result in limiting the amount of data that can be transmitted to 280 $D N$ during successive relaying phases. To closely analyze the impact 281 of the energy and data buffer capacities at the EH nodes on the overall 282 system throughput, we present the 2-D curves corresponding to the 283 individual analysis of the energy buffer size while keeping the data 284 buffer size constant, and vice versa.

The results in Fig. 4 show the throughput of the system against 286 the size of the battery in the presence of sufficient, insufficient, and 287 asymmetric data buffer sizes for both optimal and suboptimal schemes. 288 As expected, upon increasing the battery size, the throughput of the 289 system is improved, owing to the availability of increased amount of 290 


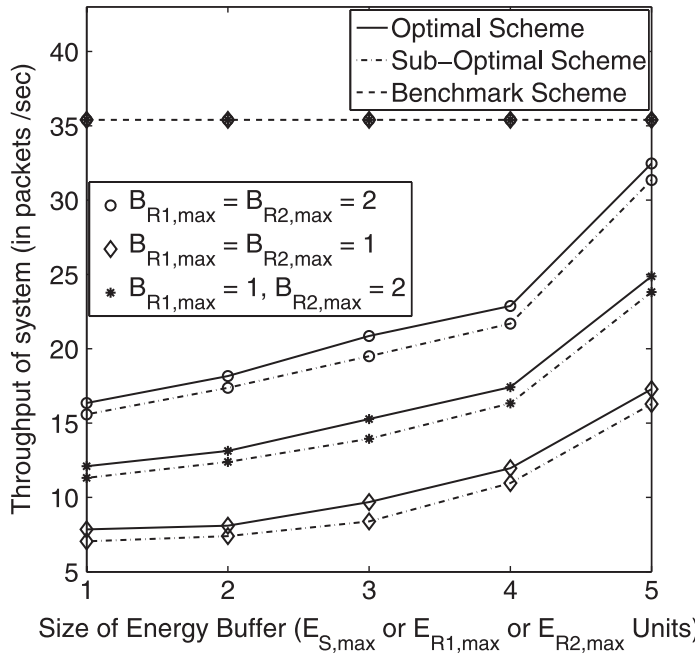

Fig. 4. Impact of energy buffer size at all the EH nodes with sufficient (two packets), insufficient (one packet), and asymmetric data buffer capacity at the RNs on the throughput of the system by the deadline $T$.

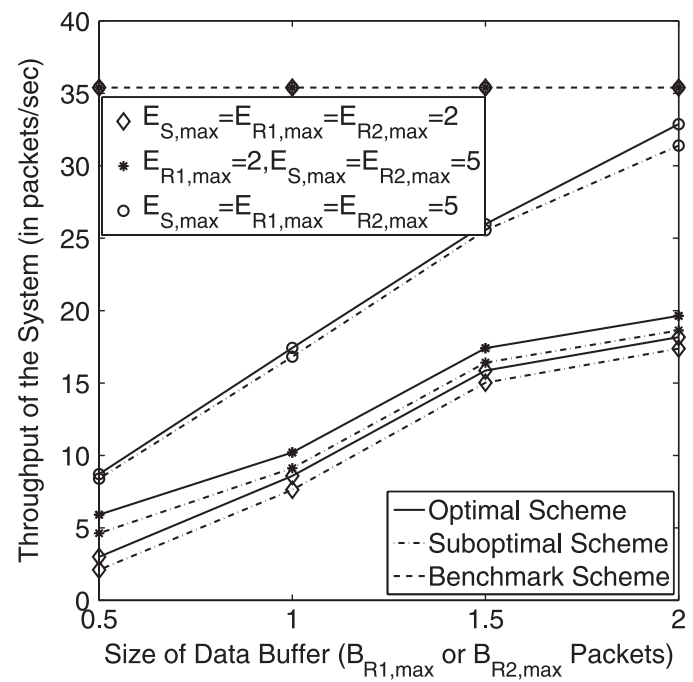

Fig. 5. Impact of data buffer size at the RNs with sufficient (five units), insufficient (two units), and asymmetric battery capacities at EH nodes over throughput of the system by the deadline $T$.

291 energy (due to the increase in buffer size) for transmission. Moreover, 292 it can be observed that for sufficient (or insufficient) data storage, 293 our optimal system is capable of achieving 92\% (or 50\%) of the 294 benchmark scheme's throughput performance [3], whereas our sub295 optimal scheme performs slightly worse than the optimal scheme, 296 reaching $88 \%$ (or $46 \%$ ) of the benchmark system's throughput value 297 in [3], when the battery capacity of the EH nodes is sufficiently high $298\left(E_{S, \max }=E_{R_{1}, \max }=E_{R_{2}, \max }=5\right.$ units $)$. Furthermore, for asym299 metric settings having unequal data buffers at $R N_{1}$ and $R N_{2}$, the 300 throughput becomes lower than that for sufficiently large storage, since $301 R N_{1}$ is now acting as a bottleneck, preventing the flow of data to $D N$. 302 On the other hand, for this asymmetric setting, the throughput becomes 303 higher than that for insufficient storage, since the node $R N_{2}$ has a 304 higher data storage capacity, thereby supporting a higher data rate to $305 D N$. The suboptimal scheme's throughput performance was $95.2 \%$, $30690.7 \%$, and $93.7 \%$ of that of the optimal scheme for the scenarios of 307 sufficient, insufficient, and asymmetric data buffers, respectively.

308 Similarly, Fig. 5 shows the throughput of the system as a function of 309 the data buffer size at the RNs with sufficient, insufficient, and asym-

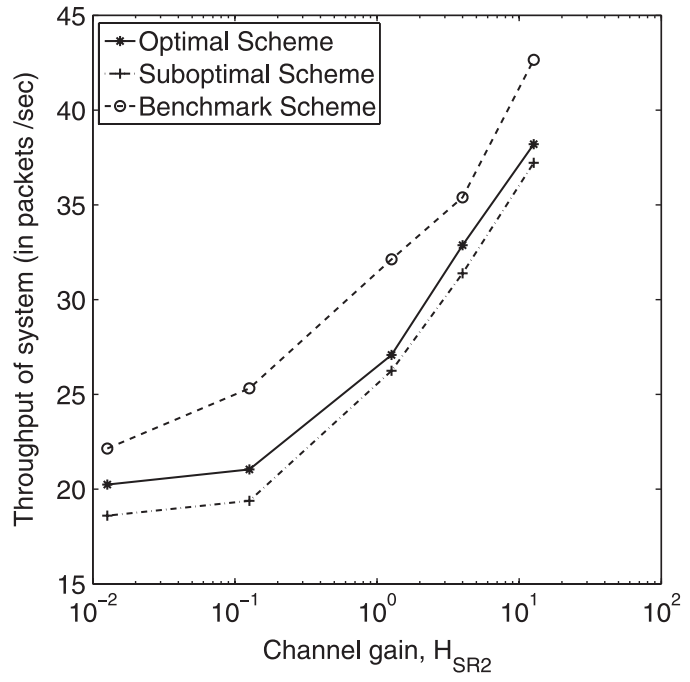

Fig. 6. Impact of asymmetric fading from $S$ to $R N_{2}$ for sufficient battery and data buffer capacities (five units and two packets, respectively) at EH nodes on throughput of the system by the deadline $T$.

metric energy buffer sizes for both optimal and suboptimal schemes. 310 It is clearly demonstrated that as the size of the data buffer increases, 311 the amount of data successfully transmitted to the $D N$ also increases 312 for both schemes, indicating that the optimal and suboptimal schemes 313 have quite similar performance. The reason behind this trend is the 314 reduction of overflowing data buffers owing to the larger capacities of 315 these buffers at the RNs. Furthermore, for sufficient (or insufficient) 316 battery capacities, our optimal system having finite buffers is capable 317 of achieving $92 \%$ (or 52\%) of the throughput compared with our 318 suboptimal scheme that performs comparably, since it achieves $88 \% 319$ (or 49\%) of the benchmark system's throughput [3] for the maximum 320 data buffer size of $B_{R_{1}, \max }=B_{R_{2} \text {, max }}=2$ packets. Furthermore, for 321 asymmetric settings having unequal energy buffers at $R N_{1}$ and $R N_{2}, 322$ the throughput becomes lower than that for a sufficiently large storage, 323 since $R N_{1}$ is low on energy, hence preventing the flow of data to $D N .324$ On the other hand, for this asymmetric setting, the throughput becomes 325 higher than that for insufficient storage, since the node $R N_{2}$ has a 326 higher energy storage capacity, consequently supporting a higher data 327 rate to $D N$. Moreover, the suboptimal scheme achieves $96.7 \%, 87.3 \%, 328$ and $94.2 \%$ of the throughput of our optimal scheme for sufficient, 329 insufficient, and asymmetric energy buffers, respectively.

330

Fig. 6 shows the throughput of the system as a function of the asym- 331 metric channel gain of the $S N-R N_{2}$ path $\left(H_{S R 2}\right)$ for the scenario of 332 having a sufficiently high data and energy buffer size at the EH nodes, 333 where all other channel gains are set to $H_{S R 1}=H_{R 1 D}=H_{R 2 D}=4.334$ It can be clearly seen that as the channel gain $H_{S R 2}$ increases, the 335 throughput of the system increases for all the schemes owing to the 336 rate-power relationship mentioned in (1). This means that as the value 337 of the channel gain increases, the amount of data transmitted from 338 $S N$ to $R N_{2}$ increases, and so does the amount of data reaching the 339 $D N$, hence, also increasing the overall throughput of the system. As 340 expected, the benchmark scheme represents the upper bound of the 341 system's throughput for an asymmetric setting of the channel gain, 342 as it relies on the idealized assumptions of infinite data and energy 343 storage capacities at the EH nodes. However, our optimal scheme 344 performs better than the suboptimal scheme owing to the fixed duration 345 of phases in the successive relaying protocol of the latter scheme. $\quad 346$

In Fig. 7, we considered the throughput of the system as a function 347 of the data buffer capacity at the RNs for the scenario of asymmetric 348 channel gains and asymmetric energy buffer capacity. Explicitly, we 349 


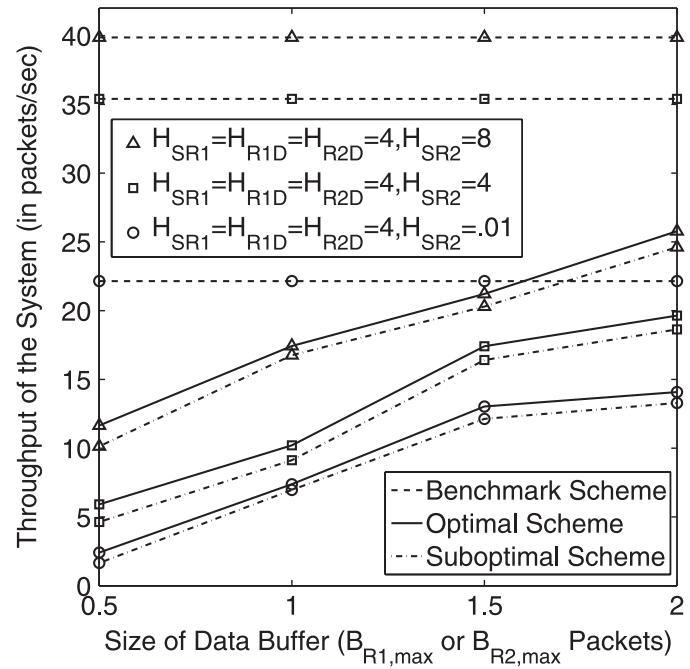

Fig. 7. Impact of data buffer size at the RNs with asymmetric channel gains and battery capacities $\left(E_{S, \max }=E_{R 2, \max }=5\right.$ units, and $E_{R 1, \max }=$ 2 units) at EH nodes over throughput of the system by the deadline $T$.

350 have used $E_{S, \max }=E_{R 2, \max }=5$ units and $E_{R 1, \max }=2$ units at the $351 \mathrm{EH}$ nodes. The benchmark scheme provides an upper bound for our 352 proposed schemes and has a constant throughput, since it is unaffected 353 by the data and energy buffer capacity at the EH nodes. Interestingly, 354 the throughput of the system improves upon increasing the value of the 355 channel gains, which becomes explicit by observing the rate-power 356 relationship of (1). Moreover, the asymmetric setting of energy buffers 357 at the $\mathrm{EH}$ nodes of the proposed scheme results in limiting the 358 throughput achieved by the system, because $R N_{1}$ is acting as the 359 bottleneck owing to the low energy buffer capacity.

360 In light of the preceding study, our findings for the realistic simula361 tion parameters in Table I may be summarized as follows.

363 1) The performance of the suboptimal scheme as a percentage of 364 the throughput achieved by the optimal scheme reaches its max365 imum when the two phases of the successive relaying protocol 366 have equal duration.

367 2) The optimal and suboptimal schemes are capable of achieving 368 up to $92 \%$ and $88 \%$ of the benchmark scheme's throughput [3] 369 for sufficiently high energy and data buffer capacities.

370 3) The suboptimal scheme's throughput is consistently about $90 \%$ 371 of that of the optimal scheme.

372 4) For asymmetric data (or energy) buffer sizes, the attainable 373 throughput depends on the total (i.e., collective) data (or energy) 374 buffer capacity available in the network and not only on the 375 smallest data buffer.

\section{CONCLUSION}

377 In this paper, we have considered the throughput optimization of an $378 \mathrm{EH}$-assisted two-hop network using a buffer-aided successive relaying 379 protocol. Under the assumption of known energy arrivals, we defined 380 the related nonconvex optimization problem and proposed both opti$381 \mathrm{mal}$ and suboptimal schemes to maximize the data delivered to the $D N$ 382 by the deadline. Then, using the interior-point method, an efficient 383 solution was found for both schemes. Finally, our results justify that 384 both our optimal and suboptimal schemes are capable of performing 385 close to the benchmark system [3]. Furthermore, the less-complex 386 suboptimal scheme is capable of approaching the performance of our 387 optimal scheme at the expense of a slight performance degradation, provided that the $\mathrm{EH}$ nodes are equipped with sufficiently large buffers 388 for both energy and data storage. Our future work may consider 389 EH-aided adaptive transceiver schemes.

\section{APPENDIX A}

\section{PROOF OF LEMMA 1}

This proof is an extension of that derived for the point-to-point case 393 in [5] to the two-hop scenario defined in this paper. Let us assume that 394 the transmitter nodes $\left(S N, R N_{1}, R N_{2}\right)$ change their transmission rate 395 between two EH instances $t_{i}$ and $t_{i+1}$. Let us furthermore denote the 396 rates by $r_{M, n}$ and $r_{M, n+1}$ and the instant when the rate changes by 397 $t_{i}^{\prime}$, where we have $M \in\{S I, R 2\}$ in Phase I and $M \in\{S I I, R 1\} 398$ in Phase II of the successive relaying protocol. Correspondingly, the 399 duration of each phase can be written as $L_{I, n}, L_{I, n+1}, L_{I I, n}$, and 400 $L_{I I, n+1}$. Let us now consider the duration $\left[t_{i}, t_{i+1}\right)$. The total energy 401 consumed in this duration at $S N$ is $p_{S I, n} L_{I, n}+p_{S I I, n} L_{I I, n}+402$ $p_{S I, n+1} L_{I, n+1}+p_{S I I, n+1} L_{I I, n+1}$. Similarly, the total energy con- 403 sumed at $R N_{1}$ is $p_{R 1, n} L_{I I, n}+p_{R 1, n+1} L_{I I, n+1}$ and that at $R N_{2}$ is 404 $p_{R 2, n} L_{I, n}+p_{R 2, n+1} L_{I, n+1}$. Let us now consider $S N$ in more detail 405 and define

$$
\begin{aligned}
p_{S I}^{\prime} & =\frac{p_{S I, n} L_{I, n}+p_{S I, n+1} L_{I, n+1}}{t_{i+1}-t_{i}} \\
p_{S I I}^{\prime} & =\frac{p_{S I I, n} L_{I I, n}+p_{S I I, n+1} L_{I I, n+1}}{t_{i+1}-t_{i}} \\
r_{S I}^{\prime} & =r\left[p_{S I}^{\prime}\right]=r\left[\frac{p_{S I, n} L_{I, n}+p_{S I, n+1} L_{I, n+1}}{t_{i+1}-t_{i}}\right] \\
r_{S I I}^{\prime} & =r\left[p_{S I I}^{\prime}\right]=r\left[\frac{p_{S I I, n} L_{I I, n}+p_{S I I, n+1} L_{I I, n+1}}{t_{i+1}-t_{i}}\right] .
\end{aligned}
$$

Let us now use these $r_{S I}^{\prime}$ and $r_{S I I}^{\prime}$ as the new transmission rates for 407 Phases I and II at $S N$ over $\left[t_{i}, t_{i+1}\right)$ and keep the rest of the rates 408 the same as in the original policy. It is easy to observe that the new 409 transmission policy is feasible, since all the energy constraints are 410 satisfied under this policy. On the other hand, we can write the total 411 number of packets that are departed from $S N$ in both of the phases 412 over this duration under this new policy as

$$
\begin{aligned}
\left(r_{S I}^{\prime}\right. & \left.+r_{S I I}^{\prime}\right)\left(t_{i+1}-t_{i}\right)=\left(r\left[p_{S I}^{\prime}\right]+r\left[p_{S I I}^{\prime}\right]\right)\left(t_{i+1}-t_{i}\right) \\
= & \left(r\left[\frac{p_{S I, n} L_{I, n}+p_{S I, n+1} L_{I, n+1}}{t_{i+1}-t_{i}}\right]\right)\left(t_{i+1}-t_{i}\right) \\
& +\left(r\left[\frac{p_{S I I, n} L_{I I, n}+p_{S I I, n+1} L_{I I, n+1}}{t_{i+1}-t_{i}}\right]\right)\left(t_{i+1}-t_{i}\right) \\
\geq & \left(r\left[p_{S I, n}\right] L_{I, n}+r\left[p_{S I, n+1}\right] L_{I, n+1}\right) \\
& +\left(r\left[p_{S I I, n}\right] L_{I I, n}+r\left[p_{S I I, n+1}\right] L_{I I, n+1}\right) \\
= & r_{S I, n} L_{I, n}+r_{S I, n+1} L_{I, n+1}+r_{S I I, n} L_{I I, n} \\
& +r_{S I I, n+1} L_{I I, n+1}
\end{aligned}
$$

where the inequality in (5b) follows from (1) in Section II, which is 414 a concave function of the transmission power $p$. Therefore, the total 415 number of packets transmitted by $S N$ in this duration under the new 416 policy is higher than those that are departed under the original policy. 417 Similarly, we can prove that the RNs under this new policy will send 418 more data to $D N$. If we keep all the rates constant, the transmissions 419 will deliver larger amounts of data to $D N$ by the deadline. This 420 contradicts the optimality of the original transmission policy. 
424 The proof derived for the two-relay case extends the single-relay 425 case of [14]. In the case of two parallel relays, we consider a feasible 426 transmission policy where one of the relays (e.g., $R N_{1}$ ) is not always 427 on, i.e., it is not transmitting or receiving data all the time. Now, if 428 we have an idle time interval right at the beginning of Phase I, we can 429 extend the epoch of $S N$ in Phase II, ensuring that there is no idle time. 430 Note that this strategy continues to satisfy all the causality and storage 431 constraints. On the other hand, if an idle time duration occurs at the 432 beginning of Phase II, we can delay the epoch of relay $R N_{1}$ without 433 violating the feasibility of our policy, because it can store more energy 434 in the meantime, and the previous argument can be used to extend the 435 epoch of $R N_{2}$ during Phase I to avoid any idle time. Similarly, we 436 can consider the scenario when $R N_{2}$ is not always on. Therefore, we 437 remove the idle times by increasing the transmission duration of one of 438 the nodes ( $S N$ or RNs) while keeping the total amount of transmitted 439 data the same. Since the rate-power relation of (1) is concave, the new 440 policy conveys the same amount of data to $D N$ while consuming less 441 energy. Hence, it is feasible. Moreover, using this proof, we can say 442 that there exists an optimal policy, where $S N$ and $D N$ are always on 443 for the twin-relay system relying on a successive relaying protocol.

\section{4 \\ 445 \\ INTERIOR-POINT OPTIMIZATION METHOD}

446 The relevant optimization techniques include IPOPT, LOQO, and 447 KNITRO [15]. The IPOPT method is more efficient than the other 448 two techniques, because it relies on tighter termination bounds and 449 utilizes comparable CPU time to evaluate a higher number of objective 450 function values and iterations [15]. The IPOPT method involves the 451 primal-dual interior-point algorithm with the aid of a so-called filter 452 line-search method invoked for nonlinear programming [15], [16], 453 which improves its robustness over that of LOQO and KNITRO. In the 454 primal-dual interior-point method, both primal and dual variables are 455 updated, whereas primal and dual iterates do not have to be feasible. 456 The search direction in this method is obtained using Newton's method 457 applied to the modified Karush-Kuhn-Tucker equations. However, the 458 basic idea behind the filter line-search algorithm involves considering 459 a trial point during the backtracking line search, where this trial point 460 is considered to be acceptable if it leads to sufficient progress toward 461 achieving the optimization goal. This algorithm maintains a "filter," 462 which is a set of values that both the objective function and the 463 constraint violation functions are prohibited from returning. For a trial 464 point to be successful, the values of the objective function and the constraint violation functions evaluated at that trial point should not 465 be a member of the filter. This filter is updated at every iteration to 466 ensure that the algorithm does not cycle in the neighborhood of the 467 previous iterate [15].

\section{REFERENCES}

[1] B. Medepally and N. Mehta, "Voluntary energy harvesting relays 470 and selection in cooperative wireless networks," IEEE Trans. Wireless 471 Commun., vol. 9, no. 11, pp. 3543-3553, Nov. 2010.

[2] I. Ahmed, A. Ikhlef, R. Schober, and R. Mallik, "Power allocation for 473 conventional and buffer-aided link adaptive relaying systems with en- 474 ergy harvesting nodes," IEEE Trans. Wireless Commun., vol. 13, no. 3, 475 pp. 1182-1195, Mar. 2014

[3] O. Orhan and E. Erkip, "Throughput maximization for energy harvesting 477 two-hop networks," in Proc. IEEE ISIT, Jul. 2013, pp. 1596-1600. 478

[4] C. Murthy and N. Mehta, "Tutorial on energy harvesting wireless com- 479 munication systems," in Proc. Nat. Conf. Commun., Kanpur, India, 480 Feb. 2014, pp. 1596-1600.

[5] J. Yang and S. Ulukus, "Optimal packet scheduling in an energy har- 482 vesting communication system," IEEE Trans. Commun., vol. 60, no. 1, 483 pp. 220-230, Jan. 2012.

[6] K. Tutuncuoglu and A. Yener, "Optimum transmission policies for battery 485 limited energy harvesting nodes," IEEE Trans. Wireless Commun., vol. 11, 486 no. 3, pp. 1180-1189, Mar. 2012.

[7] P. He, L. Zhao, S. Zhou, and Z. Niu, "Recursive waterfilling for wireless 488 links with energy harvesting transmitters," IEEE Trans. Veh. Technol., 489 vol. 63, no. 3, pp. 1232-1241, Mar. 2014.

490

[8] J. Yang, O. Ozel, and S. Ulukus, "Broadcasting with an energy harvesting 491 rechargeable transmitter," IEEE Trans. Wireless Commun., vol. 11, no. 2, 492 pp. 571-583, Feb. 2012.

[9] C.-C. Kuan, G.-Y. Lin, H.-Y. Wei, and R. Vannithamby, "Reli- 494 able multicast and broadcast mechanisms for energy-harvesting de- 495 vices," IEEE Trans. Veh. Technol., vol. 63, no. 4, pp. 1813-1826, 496 May 2014.

[10] Z. Fang, T. Song, and T. Li, "Energy harvesting for two-way OFDM 498 communications under hostile jamming," IEEE Signal Process. Lett., 499 vol. 22, no. 4, pp. 413-416, Apr. 2015.

[11] K. Tutuncuoglu, B. Varan, and A. Yener, "Optimum transmission poli- 501 cies for energy harvesting two-way relay channels," in Proc. IEEE ICC, 502 Jun. 2013, pp. 586-590.

[12] I. Ahmed, A. Ikhlef, D. Ng, and R. Schober, "Optimal resource allocation 504 for energy harvesting two-way relay systems with channel uncertainty," 505 Proc. IEEE GlobalSIP, Dec. 2013, pp. 345-348.

[13] N. Roseveare and B. Natarajan, "An alternative perspective on utility max- 507 imization in energy-harvesting wireless sensor networks," IEEE Trans. 508 Veh. Technol., vol. 63, no. 1, pp. 344-356, Jan. 2014.

[14] O. Orhan and E. Erkip, "Optimal transmission policies for energy har- 510 vesting two-hop networks," in Proc. 46th Annu. Conf. CISS, Mar. 2012, 511 pp. 1-6.

512

[15] A. Wachter and L. T. Biegler, "On the implementation of an interior-point 513 filter line-search algorithm for large-scale nonlinear programming," Math. 514 Programm., vol. 106, no. 1, pp. 25-57, Mar. 2006.

[16] S. Boyd and L. Vandenberghe, Convex Optimization. New York, NY, 516 USA: Cambridge Univ. Press, 2004. 


\section{AUTHOR QUERIES}

\section{AUTHOR PLEASE ANSWER ALL QUERIES}

AQ1 = Please provide keywords.

AQ2 = Table 1 was cited and captured as text. Please check.

END OF ALL QUERIES 